\title{
Investigation of Charge-Transfer Interactions Induced by Encapsulating Fullerene in a Mesoporous Tetrathiafulvalene-Based Metal-Organic Framework
}

Manuel Souto, Joaquín Calbo, Samuel Mañas-Valero, Aron Walsh, Guillermo Minguez Espallargas

Submitted date: 02/07/2019 - Posted date: 02/07/2019

Licence: CC BY-NC-ND 4.0

Citation information: Souto, Manuel; Calbo, Joaquín; Mañas-Valero, Samuel; Walsh, Aron; Minguez Espallargas, Guillermo (2019): Investigation of Charge-Transfer Interactions Induced by Encapsulating Fullerene in a Mesoporous Tetrathiafulvalene-Based Metal-Organic Framework. ChemRxiv. Preprint.

The design of Metal-Organic Frameworks (MOFs) incorporating electroactive guest molecules in the pores has become a subject of great interest in order to install additional electrical functionalities within the framework while maintaining porosity. In this direction, understanding the charge-transfer (CT) process between the framework and the guest molecules is crucial towards the design of new electroactive MOFs. Herein, we present the encapsulation of fullerenes $\left(\mathrm{C}_{60}\right)$ in a mesoporous tetrathiafulvalene(TTF)-based MOF. The CT process between the electron-acceptor $\mathrm{C}_{60}$ guest and the electron-donor TTF ligand is studied in detail by means of different spectroscopic techniques and density functional theory calculations. Importantly, gas sorption measurements demonstrate that sorption capacity is maintained after encapsulation of fullerenes, whereas the electrical conductivity is increased by two orders of magnitude due to the CT interactions between $\mathrm{C}_{60}$ and the TTF-based framework.

File list (2)

Manuscript.doc (6.59 MiB)

view on ChemRxiv • download file 


\section{Investigation of Charge-Transfer Interactions}

\section{Induced by Encapsulating Fullerene in a}

\section{Mesoporous Tetrathiafulvalene-based Metal-Organic}

\section{Framework}

Manuel Souto*1 ${ }^{\star 1}$ Joaquín Calbo ${ }^{2}$, Samuel Mañas-Valero $^{1}$, Aron Walsh $^{2}$ and Guillermo Mínguez Espallargas*1

Address:

${ }^{1}$ Instituto de Ciencia Molecular (ICMol), Universidad de Valencia, C/ Catedrático José Beltrán 2, 46980 Paterna, Spain and ²Department of Materials, Imperial College London, London SW7 2AZ, United Kingdom.

Email: Manuel Souto - manuel.souto@uv.es; Guillermo Mínguez Espallargas guillermo.minguez@uv.es

* Corresponding author

\section{Abstract}

The design of Metal-Organic Frameworks (MOFs) incorporating electroactive guest molecules in the pores has become a subject of great interest in order to install additional electrical functionalities within the framework while maintaining porosity. In this direction, understanding the charge-transfer (CT) process between the framework and the guest molecules is crucial towards the design of new electroactive MOFs. Herein, we present the encapsulation of fullerenes $\left(C_{60}\right)$ in a mesoporous 
tetrathiafulvalene(TTF)-based MOF. The CT process between the electron-acceptor $\mathrm{C}_{60}$ guest and the electron-donor TTF ligand is studied in detail by means of different spectroscopic techniques and density functional theory calculations. Importantly, gas sorption measurements demonstrate that sorption capacity is maintained after encapsulation of fullerenes, whereas the electrical conductivity is increased by two orders of magnitude due to the CT interactions between $\mathrm{C}_{60}$ and the TTF-based framework.

\section{Keywords}

Metal-Organic Framework; Tetrathiafulvalene; Fullerene; Donor-Acceptor; Charge Transfer

\section{Introduction}

The emerging field of Metal-Organic Frameworks (MOFs), crystalline porous materials constructed from metallic nodes and organic linkers, has been one of the major breakthroughs in Chemistry during the last decades. ${ }^{[1,2]}$ Because of their vast structural and functional possibilities, this class of hybrid materials has shown to find several applications in, for example, gas storage and separation, sensing or catalysis. $^{[3-5]}$ In addition, electroactive MOFs combining porosity and electrical conductivity $^{[6-8]}$ have also attracted large attention during the last years in view of their potential application as chemiresistive sensors, ${ }^{[9]}$ field-effect transistors ${ }^{[10]}$ or supercapacitors, ${ }^{[11]}$ among others. Whereas most of MOFs are electrical insulators, a few of such materials have shown to exhibit excellent electrical conductivity and high charge mobility based on an appropriate choice of the building units to form electrically delocalised frameworks, or by inclusion of electroactive guest molecules 
in the pores. ${ }^{[6,12-14]}$ In this direction, the incorporation of redox-active moieties ${ }^{[15-18]}$ as well as the understanding of charge-transfer (CT) processes in MOFs, ${ }^{[19-21]}$ are excellent pathways for the rational design of new electroactive frameworks exhibiting electrical conductivity and porosity at the same time.

Fullerenes $\left(\mathrm{C}_{60}\right)^{[22]}$ have demonstrated to find numerous applications in different fields, ranging from molecular electronics and nanotechnology to biomedical applications, due to their exceptional electrochemical and photophysical properties. ${ }^{[23,24]}$ In particular, understanding CT processes between electron-acceptor $\mathrm{C}_{60}$ and electron-donor molecules is fundamental for optimising photovoltaics and develop efficient solar cells. ${ }^{[25]}$ Encapsulation of $\mathrm{C}_{60}$ in $\mathrm{MOFs}{ }^{[26]}$ has become a very interesting strategy for fullerene purification, ${ }^{[27-29]}$ or to incorporate additional functionalities within the MOF. ${ }^{[30-32]}$ Very recently, Farha and co-workers have demonstrated that encapsulation of $\mathrm{C}_{60}$ in a zirconium-based MOF can lead to an enhancement of electrical conductivity due to donor-acceptor (D-A) interactions between the pyrenebased ligand (donor) and fullerene (acceptor) without significant decrease of porosity. [33]

Tetrathiafulvalene (TTF) and its numerous derivatives are redox-active electron-donor molecules with unique electronic properties that have been widely used as important building units in the field of molecular electronics as conductors, switches, sensors or rectifiers. ${ }^{[34,35]}$ Several studies have also been devoted to the development of TTF-based macrocyclic systems for their use as molecular machines or for supramolecular host-guest recognition. ${ }^{[36-38]}$ In this context, D-A interactions between $\mathrm{C}_{60}$ and discrete $\pi$-extended TTF molecules have been widely studied in solution during the last years. ${ }^{[39-44]}$ In contrast, much less is known about their supramolecular interactions in solid-state polymeric structures. 
MOFs using TTF as ligands have become an interesting new class of functional porous systems since they can incorporate additional electronic properties from TTF ligands to prepare new electrically conductive and redox-active MOFs. ${ }^{[45-48]}$ Very recently, we have reported a hierarchical and highly stable TTF-based MOF, named MUV-2, which is based on the 6-connected trimeric cluster $\left[\mathrm{Fe}_{3}\left(\mu_{3} \mathrm{O}\right)(\mathrm{COO})_{6}\right]$ as secondary building unit (SBU) and tetratopic tetrathiafulvalene-tetrabenzoic $\left(\mathrm{TTFTB}^{4-}\right)$ ligands. This MOF shows a hierarchical structure with mesoporous channels of $\sim 3 \mathrm{~nm}$ and orthogonal microporous channels of $\sim 1 \mathrm{~nm}$. In addition, it exhibits an enhanced catalytic activity for the aerobic oxidation of dibenzothiophene in diesel, ${ }^{[49]}$ and a reversible continuous breathing upon adsorption of different solvents. ${ }^{[50]}$ Importantly, the planarity of the TTF ligands can be modulated by the breathing behaviour, which directly impacts on its electrochemical properties. ${ }^{[5,51]}$ In view of the remarkable electron-donor character of the TTF-based ligands, herein we present the encapsulation of $\mathrm{C}_{60}$ in MUV-2 ( $\mathbf{C}_{60} @ \mathbf{M U V - 2}$ ) (Figure 1). A detailed study on the CT interactions between the electron-donor TTF ligands from the framework and the electron-acceptor fullerenes has been carried out by different spectroscopic techniques and theoretical calculations. Gas sorption measurements demonstrate that permanent porosity is retained, whereas electrical measurements show that conductivity is enhanced after $\mathrm{C}_{60}$ encapsulation. 


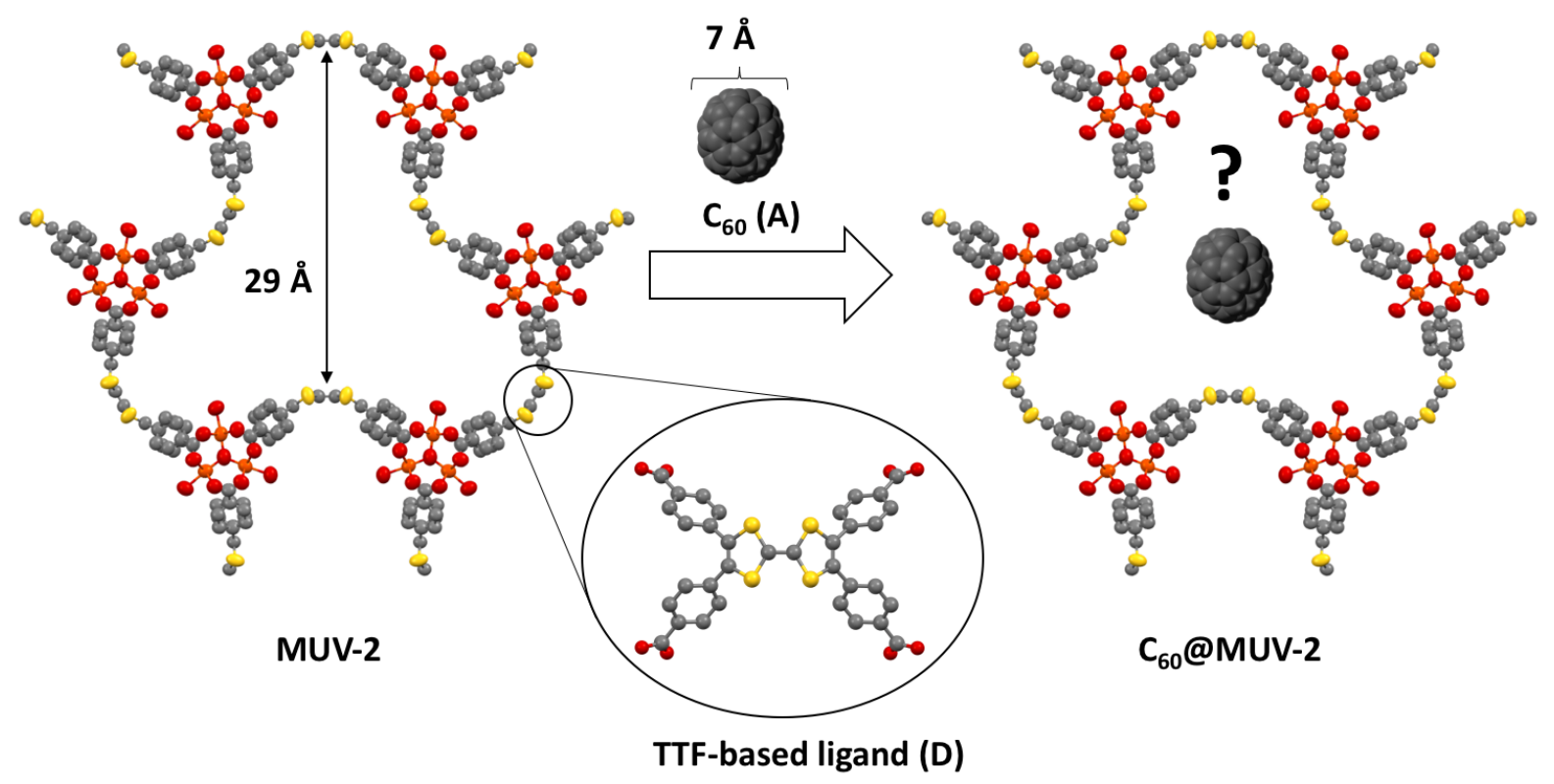

Figure 1: Schematic representation of the encapsulation of the electron-acceptor (A) $\mathrm{C}_{60}$ in the electron-donor (D) TTF-based MUV-2. Grey, yellow, orange and red spheres represent $\mathrm{C}, \mathrm{S}, \mathrm{Fe}$ and $\mathrm{O}$ atoms, respectively.

\section{Results and Discussion}

\section{Synthesis and characterisation of $\mathrm{C}_{60} @ \mathrm{MUV}-2$}

\section{Synthesis and encapsulation of $\mathrm{C}_{60}$ into MUV-2}

MUV-2 was synthesised as previously described based on the solvothermal reaction of tetrathiafulvalene tetrabenzoic acid $\left(\mathrm{H}_{4}\right.$ TTFTB), the preformed cluster $\left[\mathrm{Fe}_{3} \mathrm{O}\left(\mathrm{CH}_{3} \mathrm{COO}\right)_{6}\right] \mathrm{ClO}_{4}$ and acetic acid as modulator, in dimethylformamide (DMF). ${ }^{[49]}$ In order to activate the material, MUV-2 was exhaustively washed with DMF, methanol and heated at $150{ }^{\circ} \mathrm{C}$ for 2 hours. Encapsulation of $\mathrm{C}_{60}$ was achieved adapting a reported procedure ${ }^{[33]}$ by immersing the activated microcrystalline powder of MUV-2 in a saturated solution of $\mathrm{C}_{60}$ in $o$-dichlorobenzene for three days at $60^{\circ} \mathrm{C}$. Then, the material was washed with o-dichlorobenzene in order to remove the physisorbed $\mathrm{C}_{60}$ on the MOF surface, washed with methanol and dried at $150^{\circ} \mathrm{C}$ for 2 
hours. Powder X-ray diffraction (PXRD) pattern of $\mathbf{C}_{60} @ M U V-2$ shows that the principal peak remains at $3.4^{\circ}$ confirming that crystallinity is maintained after encapsulation of $\mathrm{C}_{60}$ and removal of the solvent (Figure 2). The needle-like morphology of $\mathbf{C}_{60} @ M U V-2$ also remained similar to the one of MUV-2 as confirmed by Scanning Electron Microscopy (SEM) (Figure S1).

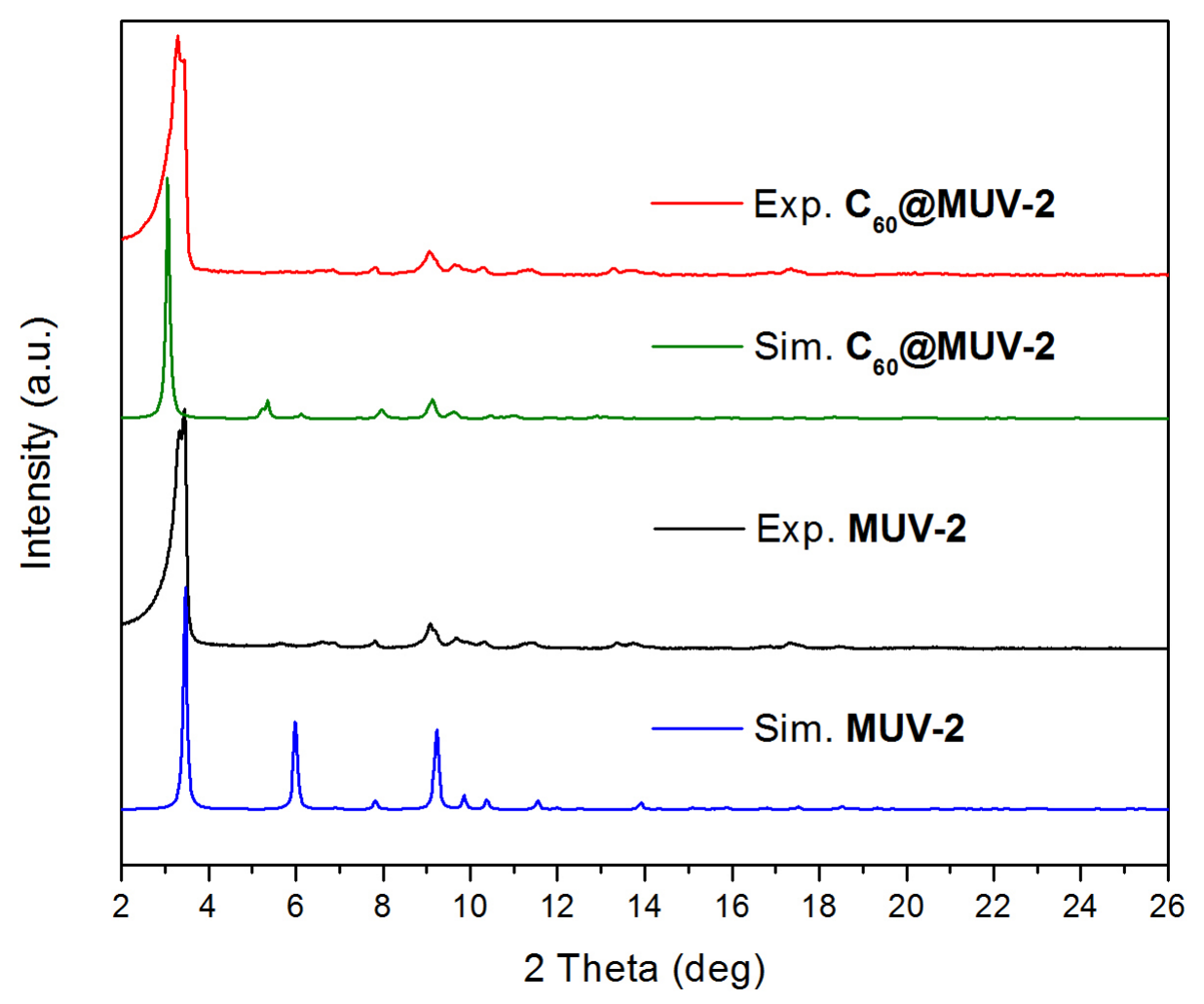

Figure 2: Powder X-ray diffraction (PXRD) patterns of simulated and experimental desolvated MUV-2 and $\mathbf{C}_{60} @ M U V-2$.

\section{Raman and UV-vis spectroscopy}

Raman spectra of $\mathbf{C}_{60}, \mathbf{M U V - 2}$ and $\mathbf{C}_{60} @ \mathbf{M U V - 2}$ crystals were measured using a Raman laser of $785 \mathrm{~nm}$ (Figure 3a). The presence of Raman bands at 218, 284 and $490 \mathrm{~cm}^{-1}$ evidences the encapsulation of $\mathrm{C}_{60}$ in MUV-2, whereas the broadening and shift of the bands towards larger frequencies are indicative of the charge-transfer 
interaction between the electron-acceptor $\mathrm{C}_{60}$ and the electron-donor TTF ligands of the framework. ${ }^{[33,52]}$ On the other hand, the UV-vis spectrum of $\mathbf{C}_{60} @ \mathbf{M U V - 2}$ crystals dispersed in $\mathrm{KBr}$ pellets (Figure $3 \mathrm{~b}$ ) shows the presence of two new bands around 260 and $350 \mathrm{~nm}$, which can be assigned to $\mathrm{C}_{60}$, whereas a broad band from 450 to $800 \mathrm{~nm}$ can be designated to an intermolecular charge-transfer (CT) excitation between the $\mathrm{C}_{60}$ and TTF ligands, as supported by theoretical calculations (see below). The energy range for the CT band is in agreement with the HOMO-LUMO gap calculated from electrochemical experiments, as the redox potential of TTF linkers can be tuned from 1.1 to $2.2 \mathrm{~V}$ (vs. $\mathrm{Ag} / \mathrm{AgCl})^{[50]}$ depending on the planarity of the ligands and the redox potential of $\mathrm{C}_{60}$ is $-0.33 \mathrm{~V}(\mathrm{vs}$. $\mathrm{Ag} / \mathrm{AgCl})$, thus the $\mathrm{CT}$ band should appear between 1.4-2.5 eV (495-885 nm).
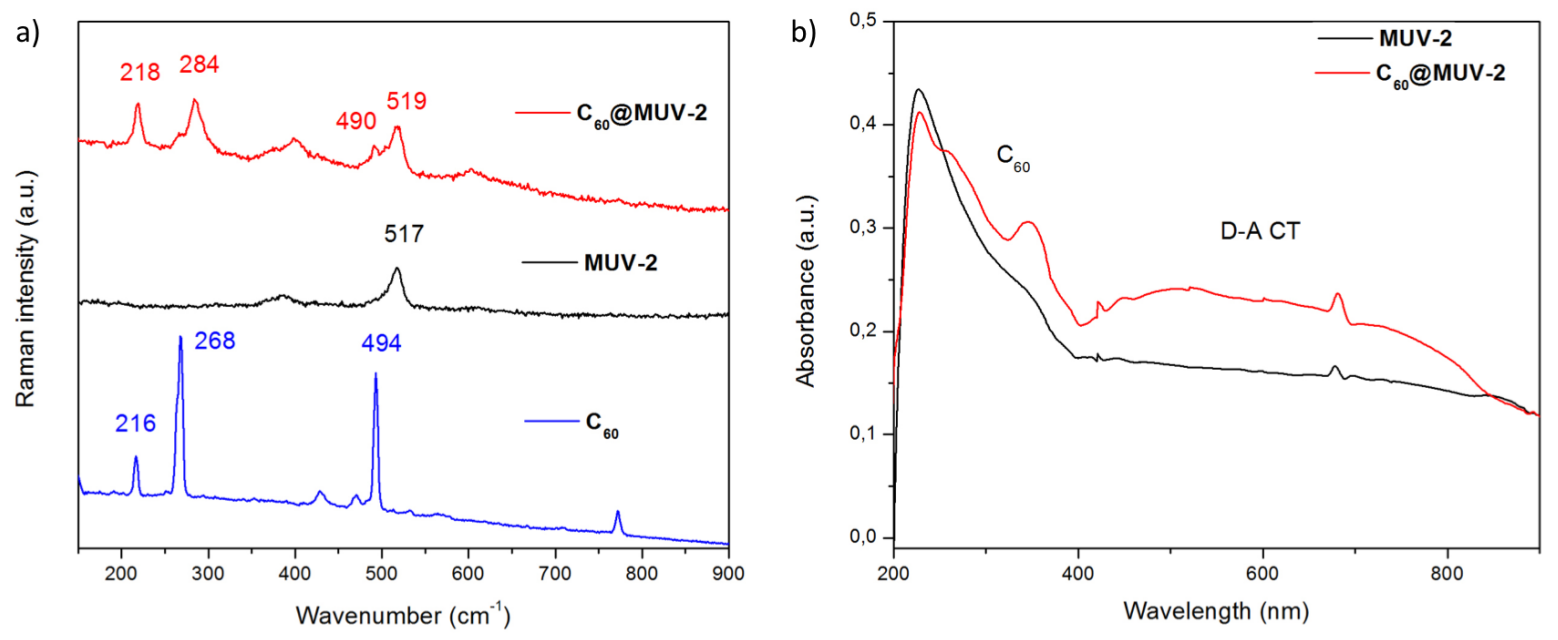

Figure 3: a) Raman spectra of $\mathbf{C}_{60}, \mathbf{M U V - 2}$ and $\mathbf{C}_{60} @ \mathbf{M U V - 2}$. b) Solid-state UV-vis spectra of MUV-2 and $\mathbf{C}_{60} @ M U V-2$. The spectra were recorded by dispersing the samples in $\mathrm{KBr}$ pellets.

\section{Gas sorption measurements}

The porosity of $\mathrm{C}_{60} @ \mathrm{MUV}-2$ was studied by means of $\mathrm{N}_{2}$ and $\mathrm{CO}_{2}$ isotherms measurements (Figure 4). Nitrogen adsorption isotherm at $77 \mathrm{~K}$ showed a combination of Types I and IV isotherms (Figure 4a), as in the case of MUV-2, 
indicating the presence of micropores and mesopores in the framework. $\mathbf{C}_{60} @ \mathbf{M U V - 2}$ has a Brunauer-Emmett-Teller (BET) surface area of $1040 \mathrm{~m}^{2} / \mathrm{g}$, which is slightly lower than that of MUV-2 $\left(1190 \mathrm{~m}^{2} / \mathrm{g}\right)$, thus confirming that porosity is retained after encapsulation of $\mathrm{C}_{60}$, in agreement with other reported examples. ${ }^{[33,53]}$ The pore volume decreased from $0.53 \mathrm{~cm}^{3} / \mathrm{g}$ to $0.44 \mathrm{~cm}^{3} / \mathrm{g}$ after encapsulation of $\mathrm{C}_{60}$ in $\mathbf{M U V - 2}$, whereas the average pore diameter calculated by means of the Barret-JoynerHalenda (BJH) method was found to be similar in both cases $(\sim 35 \AA)$. The quantity of fullerene encapsulated in MUV-2 was estimated from the decrease in pore volume, obtaining a value of around $0.7 \mathrm{C}_{60}$ per 3 TTF ligands, almost 1 fullerene per section of the void. This low encapsulation can be explained by diffusion issues or by weak interactions between the $\mathrm{C}_{60}$ and the framework, which are not strong enough to keep the $\mathrm{C}_{60}$ retained during the washing procedure. On the other hand, $\mathrm{CO}_{2}$ isotherm on $\mathbf{C}_{60} @ M U V-2$ at $298 \mathrm{~K}$ also showed a small decrease in the gas sorption capacity (Figure $4 \mathrm{~b})$, especially at high pressures $\left(7.7\right.$ and $5.3 \mathrm{mmol} \mathrm{CO}_{2} / \mathrm{g}$ at 18 bar for MUV2 and $\mathrm{C}_{60} @ \mathrm{MUV-2}$, respectively).
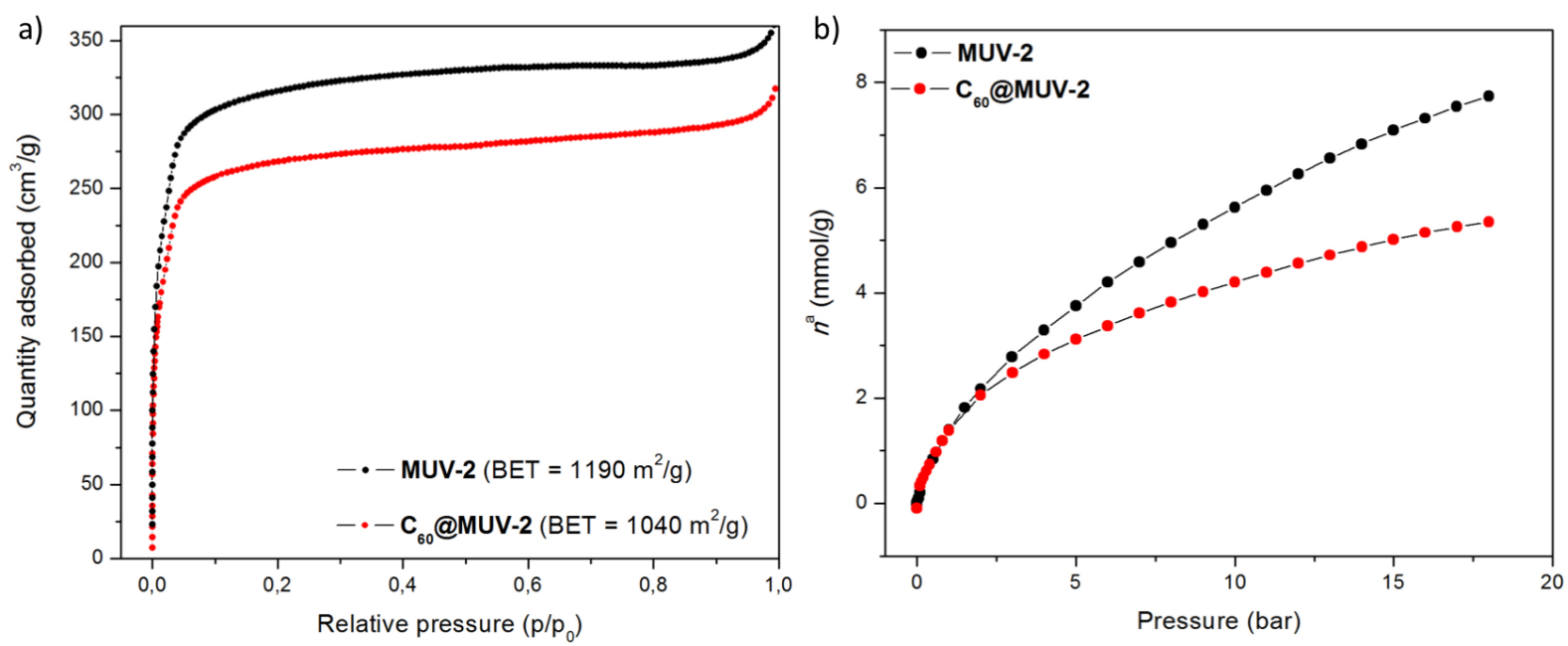

Figure 4: a) Nitrogen adsorption isotherms at $77 \mathrm{~K}$ and b) high-pressure $\mathrm{CO}_{2}$ adsorption isotherms at $298 \mathrm{~K}$, on MUV-2 (black) and $\mathbf{C}_{60} @ \mathbf{M U V - 2}$ (red). 


\section{Theoretical calculations}

In order to get further insight into the donor-acceptor interactions between $\mathbf{C}_{60}$ and the TTF-based MOF, theoretical calculations were performed under the density functional theory (DFT). The MUV-2 framework was modelled as previously described, ${ }^{[50]}$ with a high-spin $\mathrm{Fe}(\mathrm{III})$ configuration and one fullerene $\mathrm{C}_{60}$ guest molecule per pore (according to the experimental encapsulation efficiency). The hostguest $\mathbf{C}_{60} @$ MUV-2 system was fully optimized under periodic boundary conditions using the PBEsol functional with dispersion corrections (see the Experimental section for details). We initially modelled the fullerene $C_{60}$ guest in the middle of the MUV-2 mesopore. After several relaxation steps, the $\mathrm{C}_{60}$ was able to accommodate in one of the three cavities to interact favourably with the TTF-based ligand. We explored two possible conformations for the host-guest $\mathbf{C}_{60} @$ MUV-2 system (A and B; Figure 5a). In conformer A, the fullerene ball remains in the void between two TTFTB ligands, approaching one of them with short $\mathrm{C}\left(\mathrm{C}_{60}\right) \cdots \mathrm{S}($ TTFTB $)$ and $\mathrm{C}\left(\mathrm{C}_{60}\right) \cdots$ benzene(TTFTB) contacts calculated at 3.4 and $3.5 \AA$, respectively (Figure 5b). In conformer B, fullerene remains over the TTFTB ligand, promoting an efficient concave-convex complementarity with a large amount of noncovalent interactions between the $C_{60}$ ball

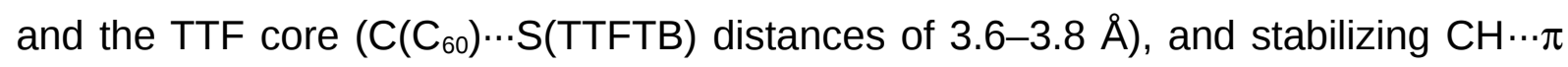
contacts between the benzene rings of TTFTB and the fullerene (2.5 $\AA$, Figure 5). Analysis of the $\mathrm{NCl}$ index allows visualization of the noncovalent interactions between the TTFTB ligand and the $\mathrm{C}_{60}$ guest, showing a significantly larger $\mathrm{NCl}$ surface for conformer B compared to A (Figure 5b). 
a)
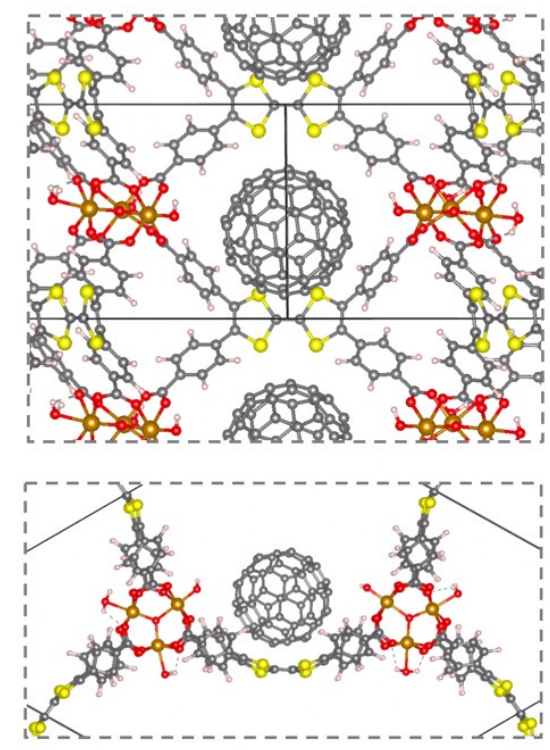

b)

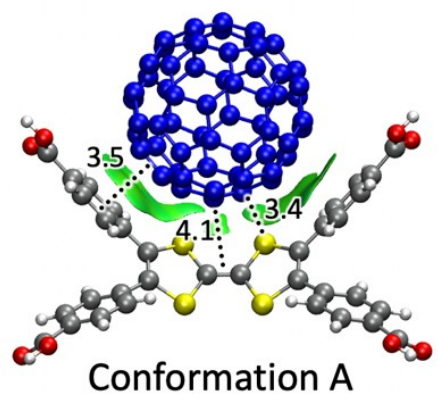

Conformation B
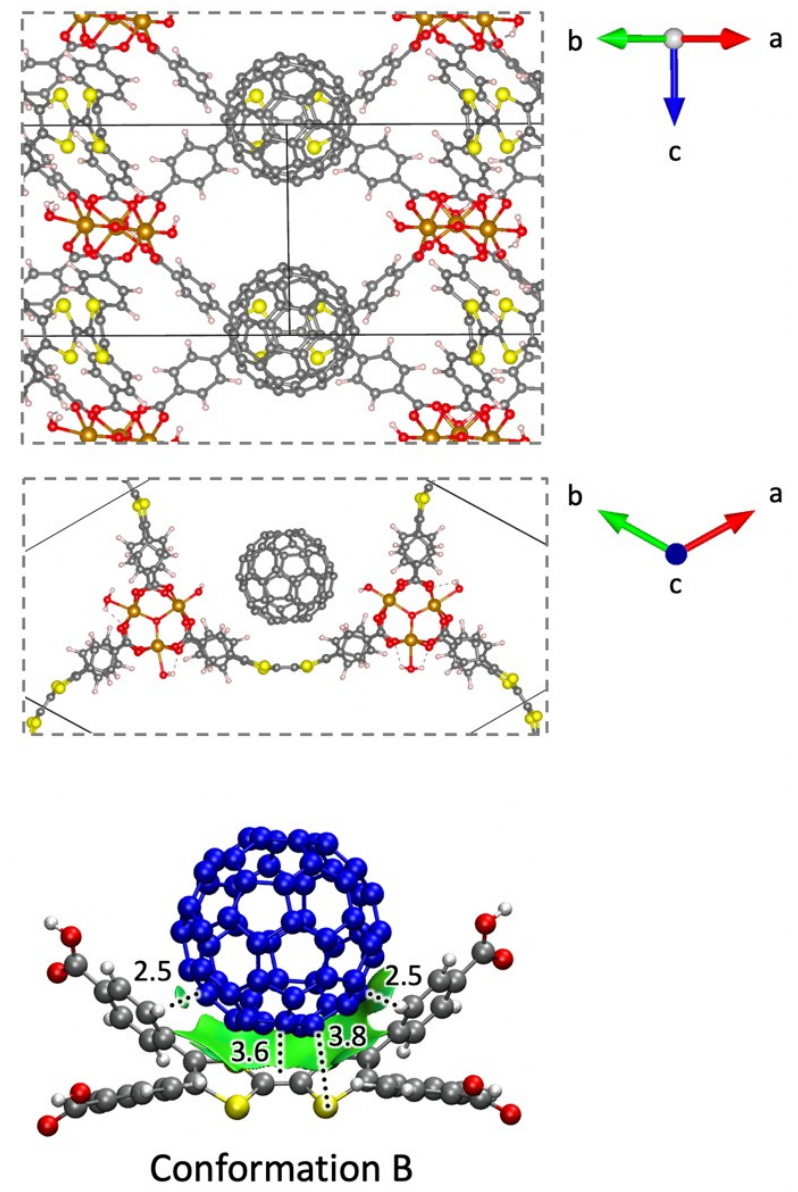

Figure 5: a) Minimum-energy crystal structure calculated for conformations $A$ and $B$ of host-guest $\mathbf{C}_{60} @ M U V-2$ at the PBEsol level in periodic boundary conditions (PBC). b) Supramolecular assemblies of $\mathbf{C}_{60} @$ TTFTB extracted from the PBC-optimized $\mathrm{C}_{60} @ \mathrm{MUV}-2$ system in arrangements A and B. Intermolecular short contacts (in Å) and $\mathrm{NCl}$ surfaces (reduced density gradient isovalue $=0.3$ a.u.) are displayed. Interaction energies $\left(E_{\text {int }}\right)$ of -20.01 and $-23.74 \mathrm{kcal} / \mathrm{mol}$ are calculated for $\mathrm{C}_{60} @$ TTFTB in conformer $\mathrm{A}$ and $\mathrm{B}$, respectively. Fullerene $\mathrm{C}_{60}$ is coloured in blue for better viewing.

Accurate hybrid DFT molecular calculations including dispersion corrections were performed to quantify the total stabilization gained when $\mathrm{C}_{60}$ interacts with MUV-2 in arrangements $\mathrm{A}$ and $\mathrm{B}$. Interaction energies $\left(E_{\mathrm{int}}\right)$ were calculated for the cluster 
$\mathrm{C}_{60} @$ TTFTB at the B3LYP-D3/6-31G** level of theory with counterpoise correction, using the minimum-energy geometry previously obtained under periodic boundary conditions (see Experimental section). Theoretical calculations indicate that $\mathrm{C}_{60}$ favourably interacts with the TTFTB ligand, with large $E_{\text {int }}<-20 \mathrm{kcal} / \mathrm{mol}$ in both arrangements. Conformer $\mathrm{B}$, in which the fullerene is placed over the TTF moiety promoting an efficient concave-convex complementarity (Figure 5), is predicted the most stable arrangement, with an $E_{\text {int }}$ of $-23.74 \mathrm{kcal} / \mathrm{mol}$ (ca. $4 \mathrm{kcal} / \mathrm{mol}$ more stable than conformer A). Henceforth, we focus subsequent analysis on conformation B.

Electronic structure calculations indicate that $\mathbf{C}_{60} @ M U V-2$ presents a small bandgap calculated to be $0.90 \mathrm{eV}$ in spin-up or $\alpha$-channel, and $0.72 \mathrm{eV}$ in spin-down or $\beta$ channel (Figure 6) - slightly smaller than that predicted for pristine MUV-2 $(0.86 \mathrm{eV}$ in $\beta$-channel). ${ }^{[50]}$ Analysis of the projected density of states (PDoS) indicates that the valence band maximum (VBM) in $\mathbf{C}_{60} @ M U V-2$ corresponds to the electron-rich TTF unit (Figure 6a). The highest-occupied crystal orbital (HOCO) displays the typical shape of the TTF HOMO and confirms the TTF-nature of the VBM (Figure 6b). In the $\alpha$-channel, the conduction band minimum (CBM) is described by the fullerene moiety, being the lowest-unoccupied crystal orbital (LUCO) completely localized on the $\mathrm{C}_{60}$ ball. Otherwise, the CBM in the $\beta$-channel is best described by the unoccupied Fe $d$ orbitals of the inorganic cluster of the MOF, the eigenstates corresponding to the fullerene being only $0.2 \mathrm{eV}$ above in energy (Figure 6). Due to the relatively low bandgap, the nature of the frontier crystal orbitals and the close proximity between the electroactive donor TTF and acceptor $\mathrm{C}_{60}$ moieties, charge-transfer processes are expected upon light irradiation. 
a)

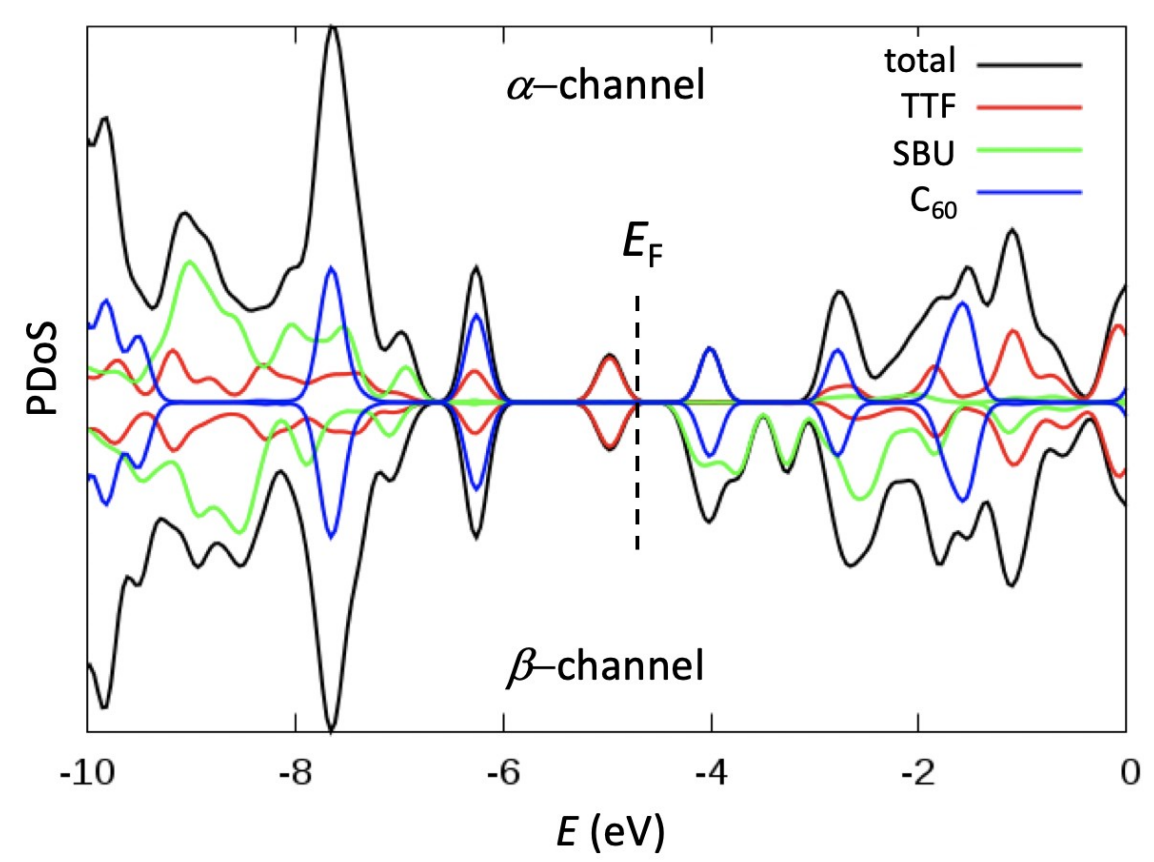

b)
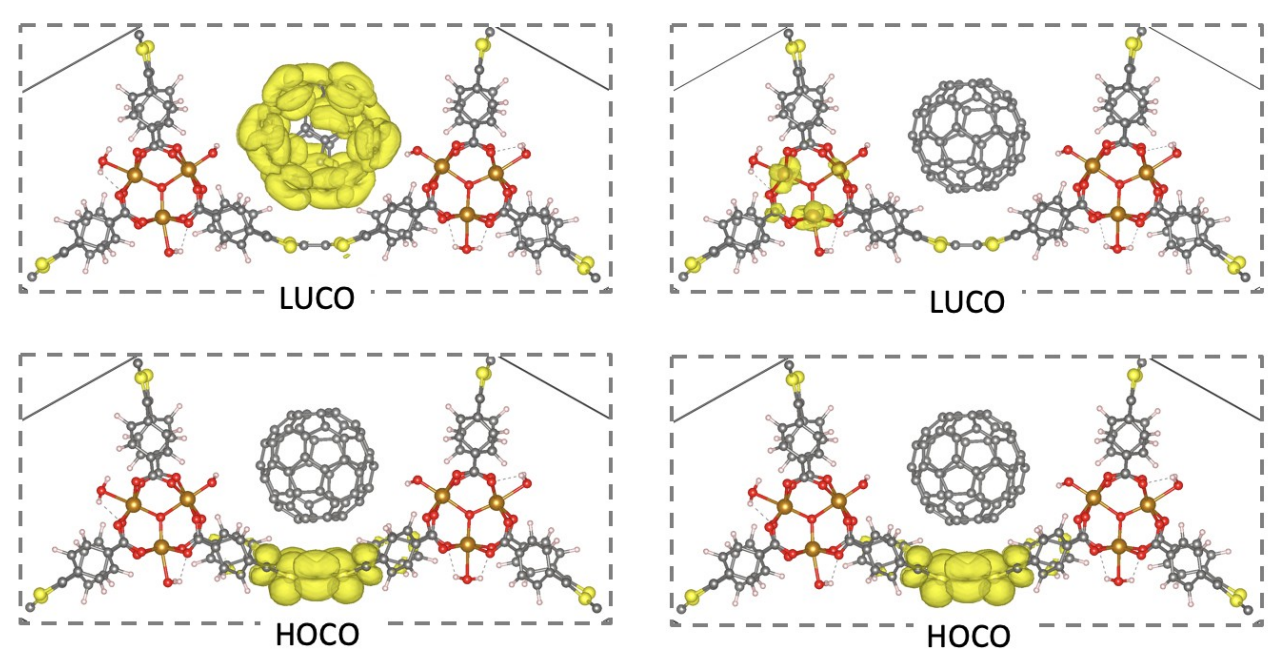

$\alpha$-channel

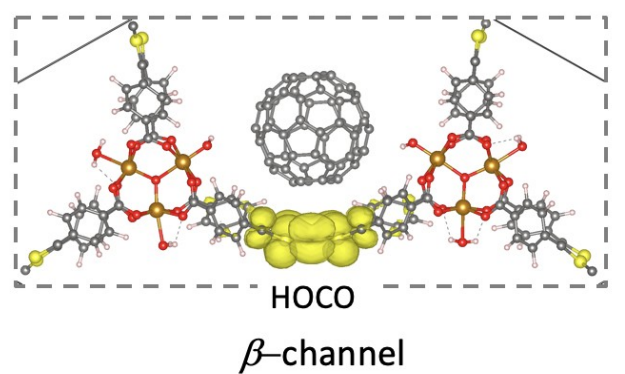

Figure 6: a) Projected density of states (PDoS) for the host-guest $\mathbf{C}_{60} @ \mathbf{M U V - 2}$ system, with contributions from the TTF core, the secondary building unit (SBU), and the fullerene $\mathrm{C}_{60}$. The Fermi level $\left(E_{F}\right)$ is indicated, and the energy reference is set to vacuum. b) Highest-occupied (HOCO) and lowest-unoccupied (LUCO) crystal orbitals in the two spin channels for $\mathbf{C}_{60} @ M U V-2$.

Donor-acceptor interactions in $\mathbf{C}_{60} @ \mathbf{M U V - 2}$ were first assessed at the ground state electronic configuration. The electron density difference between the framework 
interacting with $\mathrm{C}_{60}\left(\mathbf{C}_{60} @ \mathbf{M U V}-2\right)$ and the individual moieties $\left(\mathbf{C}_{60}+\mathbf{M U V - 2}\right)$ suggests a partial charge transfer from the TTF to the fullerene ball. Blue regions in Figure 7 indicate that the electron density is depleted from the TTF unit, especially from the $S$ lone pairs, and is accumulated (yellow volumes) in the fullerene regions close to the TTFTB ligand. The partial charge transfer from the donor TTFTB ligand to the acceptor fullerene moiety in the ground state is calculated to be as small as $0.02 \mathrm{e}$, with an exponential decay as a function of the $\mathrm{C}_{60} \ldots$ TTF intermolecular distance (Table S1).

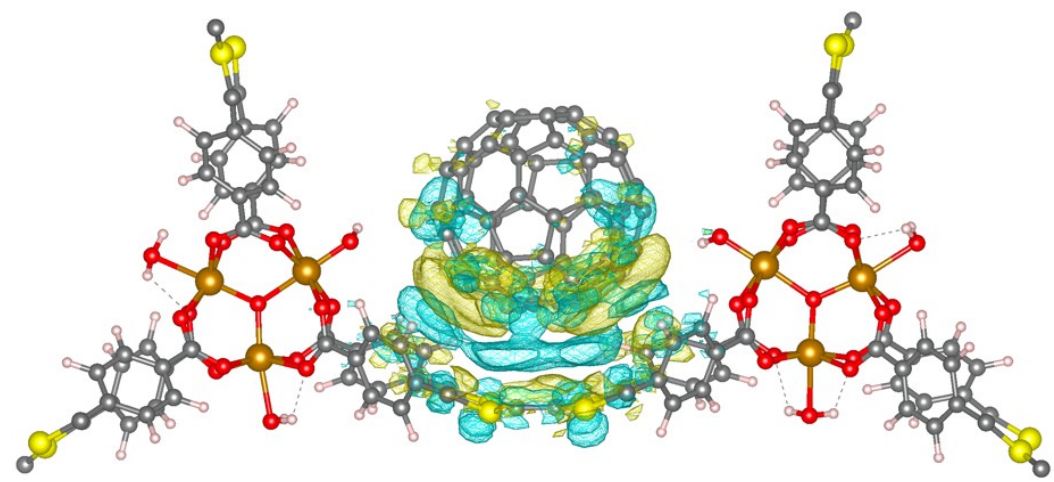

Figure 7: Electron density difference between host-guest $\mathbf{C}_{60} @ \mathbf{M U V - 2}$ and the constituting moieties $\left(\mathbf{C}_{60}+\mathbf{M U V - 2}\right)$. Blue and yellow regions indicate charge depletion and accumulation, respectively.

Time-dependent DFT molecular calculations were performed at the CAM-B3LYP/6$31 G^{* *}$ level (see the Experimental section) to shed light into the photoinduced charge-transfer process of $\mathbf{C}_{60} @ \mathbf{M U V - 2}$. Figure 8 displays the simulated absorption spectra predicted for the cluster $\mathbf{C}_{60} @$ TTFTB system in the most stable arrangement $\mathrm{B}$, the TTFTB ligand, and the fullerene guest (the triplet excitation energies are indicated). The high-energy region (<400 nm) of the experimental UV-vis absorption spectrum of $\mathbf{C}_{60} @$ MUV-2 is dominated by the MUV-2 framework (Figure 3b). Theoretical calculations predict several intense transitions in the $<300 \mathrm{~nm}$ region for 
TTFTB ligand (Table S2) that explain the experimental wide band with maximum at ca. 230 nm recorded for MUV-2 and $\mathbf{C}_{60} @$ MUV-2. These transitions are described by $\pi-\pi^{\star}$ electronic promotions involving the TTF and the peripheral carboxybenzene groups $\left(\mathrm{S}_{6}, \mathrm{~S}_{8}, \mathrm{~S}_{21} \mathrm{~S}_{54}\right)$ in the TTFTB ligand (Table $\mathrm{S} 2$ ). Singlet excited states $\mathrm{S}_{1}$ and $\mathrm{S}_{3}$ are predicted with less intensity (oscillator strength $f<0.2$ ), are described by TTF $\rightarrow$ benzene and TTF-centred monoexcitations, respectively, and give rise to the shoulder experimentally recorded at ca. 350 nm for $\mathbf{C}_{60} @ \mathbf{M U V - 2}$ (Table S2). On the other hand, the predicted singlet excited states of fullerene $S_{37}-S_{39}(f \approx 0.2)$ and $S_{52}-$ $\mathrm{S}_{54}(f \approx 0.1)$ in the region of 280 and $260 \mathrm{~nm}$ (Table S2), respectively, correlate with the experimental features that appear at $325 \mathrm{~nm}$ and $275 \mathrm{~nm}$ in host-guest C60@MUV-2 (Figure 3b).

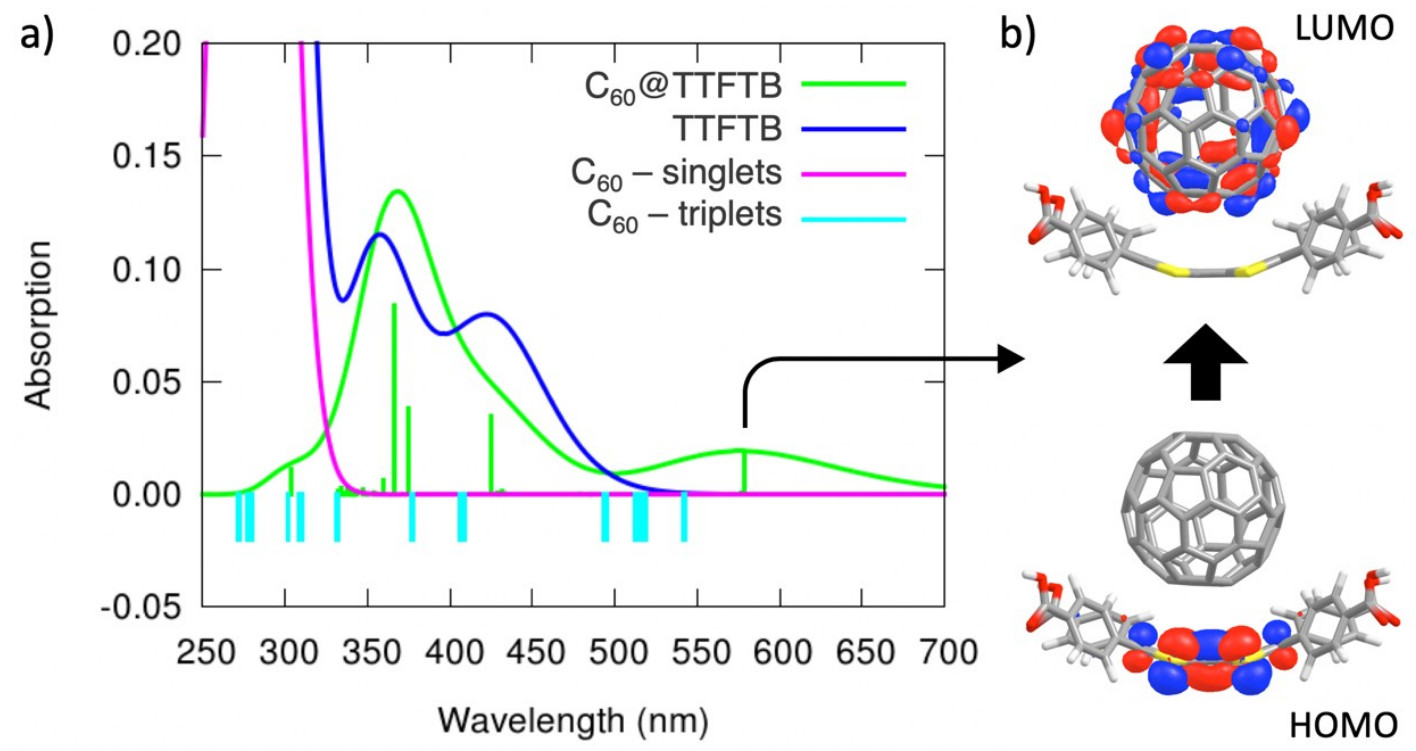

Figure 8: a) TDDFT absorption spectra calculated for host-guest $\mathbf{C}_{60} @ T T F T B$ (including vertical excitation energies), TTFTB ligand, and fullerene $\mathrm{C}_{60}$ (singlets and triplet energies). b) Monoelectronic excitation that describes the nature of the lowestlying singlet excited state $\mathrm{S}_{1}$ of $\mathbf{C}_{60} @ T T F T B$. 
Importantly, a new singlet excitation is computed for $\mathbf{C}_{60} @$ TTFTB $\left(\mathrm{S}_{1}\right)$ at 578 nm, which is not predicted either for the TTFTB ligand or the fullerene $\mathrm{C}_{60}$ (Figure 8). This transition has relatively small intensity $(f=0.018)$, and can be described by oneelectron promotion from the TTF to the $C_{60}$, i.e. it has a charge-transfer (CT) nature (Figure 8b). The position of this CT excitation (578 nm) nicely agrees with the lowenergy broad band that appears in the experimental absorption spectrum of $\mathbf{C}_{60} @$ MUV-2, centred at $550 \mathrm{~nm}$ and expanding up to $800 \mathrm{~nm}$. In fact, theoretical calculations indicate that the intensity and energy of the $S_{1} C T$ transition in $\mathbf{C}_{60} @$ TTFTB is significantly affected by the intermolecular TTF... $\mathbf{C}_{60}$ distance and the characteristic TTF boat dihedral angle (Table S3). The charge transfer from the TTF to the fullerene ball in the $\mathrm{S}_{1} \mathrm{CT}$ excitation of $\mathbf{C}_{60} @$ TTFTB is calculated to be of nearly 1 electron (0.94e at the minimum-energy geometry, Table S1).

\section{Electrical measurements}

In order to analyse the possible enhancement of electrical conductivity after encapsulation of $\mathrm{C}_{60}$ in $\mathbf{M U V - 2}$, transport measurements for MUV-2 and $\mathbf{C}_{60} @ \mathbf{M U V - 2}$ were performed using two-contact probe pressed-pellet devices measured at room temperature $(300 \mathrm{~K})$ (Figure 9). Interestingly, pellet of $\mathbf{C}_{60} @ \mathbf{M U V - 2}$ shows an increase of around two orders of magnitude $\left(\sigma=4.7 \cdot 10^{-9} \mathrm{~S} / \mathrm{cm}\right)$ compared to the very resistive MUV-2 $\left(\sigma=3.7 \cdot 10^{-11} \mathrm{~S} / \mathrm{cm}\right.$, Table 1$)$. This enhancement of the

electrical conductivity can be explained by the D-A charge transfer from the TTF linkers to the $\mathrm{C}_{60}$ since the fullerene is acting as a dopant introducing charge carriers within the framework. However, this enhancement in conductivity is lower in comparison to other reported systems ${ }^{[14,32]}$ probably due to the low ratio between $\mathrm{C}_{60}$ 
and TTF (1:4) and the long distances between the TTF moieties (9.6 $\AA$ along the $c$ axis), which could prevent the charge delocalisation along the framework.

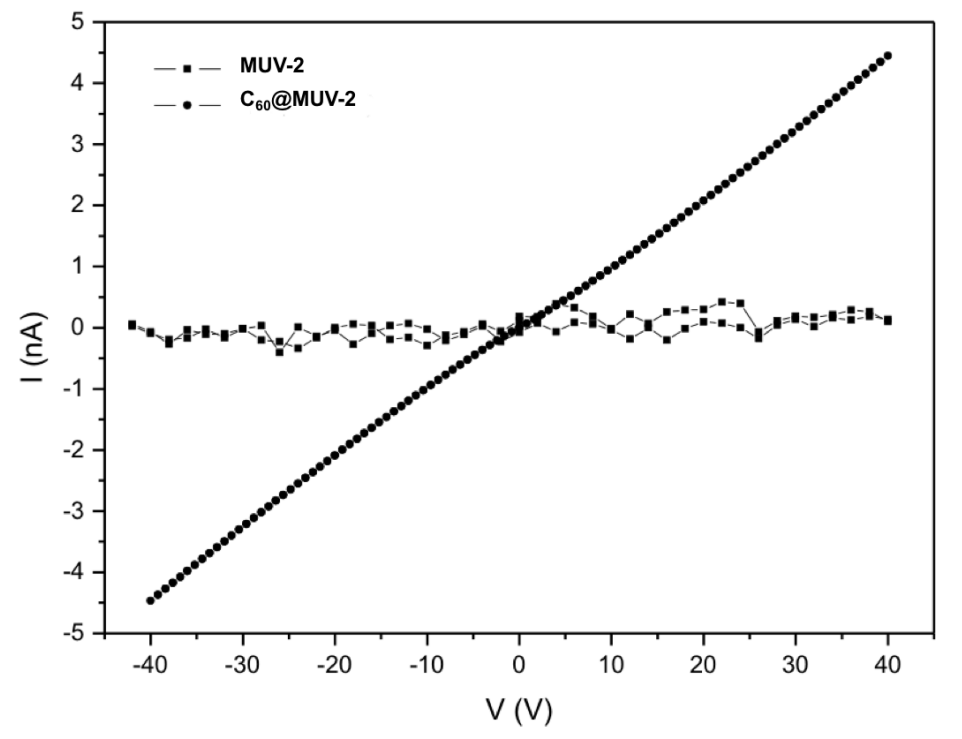

Figure 9: Current (I) - Voltage (V) plot for pressed pellets of MUV-2 and $\mathbf{C}_{60} @ \mathbf{M U V - 2}$ at $300 \mathrm{~K}$.

Table 1: Geometrical factors (length I, width w, and thickness t), resistance obtained by the linear fit of the ohmic regime of the I-V curves and conductivity for MUV-2 and $\mathbf{C}_{60} @ M U V-2$, at $300 \mathrm{~K}$.

\begin{tabular}{|c|c|c|c|c|c|}
\hline Sample & $\mathbf{I}(\mu \mathrm{m})$ & $\mathbf{w}(\mu \mathrm{m})$ & $\mathbf{t}(\boldsymbol{\mu m})$ & $\mathbf{R}(\boldsymbol{\Omega})$ at $\mathbf{3 0 0} \mathrm{K}$ & $\sigma(\mathbf{S} / \mathbf{c m})$ at 300 K \\
\hline MUV-2 & 110 & 480 & 65 & $(9.5 \pm 0.6) \cdot 10^{11}$ & $3.7 \cdot 10^{-11}$ \\
\hline $\mathrm{C}_{60} @ M U V-2$ & 325 & 540 & 150 & $(8.510 \pm 0.015) \cdot 10^{9}$ & $4.7 \cdot 10^{-9}$ \\
\hline
\end{tabular}

\section{Conclusion}

In summary, we have reported the encapsulation of $\mathrm{C}_{60}$ in a mesoporous TTF-based MOF (MUV-2). Charge-transfer interactions between $\mathrm{C}_{60}$ and TTF ligands from the framework in $\mathbf{C}_{60} @ M U V-2$ were confirmed by different spectroscopic techniques and theoretical calculations. Interestingly, after encapsulation of fullerene, gas sorption measurements demonstrated that mesoporosity of the MOF is maintained, and 
electrical measurements revealed an increase of around 2 orders of magnitude in conductivity, which can be explained by charge-transfer donor-acceptor (TTF $\rightarrow \mathrm{C}_{60}$ ) interactions. Current research is focused on the improvement of electrical conductivity in MUV-2 and the photophysical characterisation of the charge transfer in $\mathbf{C}_{60} @ M U V-2$.

\section{Experimental}

General methods and materials. All reagents and solvents employed for the syntheses were of high purity grade and were purchased from Sigma-Aldrich Co., and $\mathrm{TCl}$. Powder X-ray diffraction spectra were recorded using $0.7 \mathrm{~mm}$ borosilicate capillaries that were aligned on an Empyrean PANalytical powder diffractometer, using $\mathrm{Cu} \mathrm{K} \alpha$ radiation $(\lambda=1.54056 \AA)$. Raman spectra were acquired with a microRaman (model XploRA ONE from Horiba, Kyoto, Japan) with a grating of $1200 \mathrm{gr} / \mathrm{mm}$ and a wavelength of $785 \mathrm{~nm}$. UV-vis absorption spectra were recorded on a Jasco V670 spectrophotometer in baseline mode from 400 to $800 \mathrm{~nm}$ range. The absorption spectra were measured on the solid state by dispersed the crystals on $\mathrm{KBr}$ pellets. Nitrogen isotherms were measured using a TriStar II PLUS apparatus (Micromeritics) at $77 \mathrm{~K}$. The BET surface area was calculated by using the Brunauer-Emmett-Teller equation. The high-pressure $\mathrm{CO}_{2}$ adsorption isotherms were measured in a IGA-100 gravimetric sorption analyzer (Hiden Isochema) and the sample was degassed for 2 hours at $150^{\circ} \mathrm{C}$ under vacuum.

Synthesis of MUV-2 and encapsulation of $\mathbf{C}_{60}$. MUV-2 was synthesised and characterised as previously reported. ${ }^{[49]}$ Then $30 \mathrm{mg}$ of fullerene $\left(\mathrm{C}_{60}\right)$ was dissolved in $2 \mathrm{~mL}$ of $\mathrm{o}$-dichlorobenzene and activated MUV-2 $(10 \mathrm{mg})$ was added to it. The vial 
was heated at $60{ }^{\circ} \mathrm{C}$ for 3 days and the MOF was then exhaustively washed with 0 dichlorobenzene to remove any physisorbed $\mathrm{C}_{60}$ on the MOF surface, washed with methanol and finally dried at $150{ }^{\circ} \mathrm{C}$ for 2 hours.

Computational Details. Theoretical calculations were performed under the density functional theory framework. Periodic boundary conditions (PBC) calculations were carried out with the FHI-AIMS (Version 171221) software. ${ }^{[54]}$ MUV-2 was modelled as previously described, with $\mathrm{Fe}(\mathrm{III})$ ions in a high-spin $d^{5}$ configuration. Guest $\mathrm{C}_{60}$ molecule was rationally inserted into the bigger mesoporous channel of MUV-2 in the most plausible sites, and the geometry of the host-guest $\mathbf{C}_{60} @ \mathbf{M U V - 2}$ system was fully relaxed at the PBEsol functional ${ }^{[55]}$ with tier 1 basis set. Dispersion corrections were added according to the Hirshfeld partitioning of the electron density (Tkatchenko-Scheffler method). ${ }^{[56]}$ Electronic structure calculations were performed for band structure analysis using the hybrid HSE06 functional[ ${ }^{[57]}$ and tier 1 basis set. Energy reference was set to vacuum according to the protocol reported by Butler et al. ${ }^{[58]}$ Crystal structures, crystal orbitals and electron density differences were plotted by means of VESTA (version 3.4.6) software. ${ }^{[59]}$ NonCovalent Index (NCI) calculations were performed under the NCIPLOT-3.0 software ${ }^{[60,61]}$ using the default PROMOLECULAR atomic densities, and density and gradient thresholds. The intermolecular contribution to the $\mathrm{NCl}$ surfaces was calculated by means of the INTERMOLECULAR keyword, and the VMD-1.9.3 software ${ }^{[62]}$ was employed for graphical display. Molecular DFT calculations were performed for the $\mathbf{C}_{60} @$ TTFTB system using the Gaussian-16.A03 suite of packages. ${ }^{[63]}$ Hydrogen atoms were added in the terminal carboxylate groups for charge neutrality. Interaction energies were calculated for the previously PBC-optimized crystal structures as the energy difference between the dimer and the constituting monomers. The hybrid B3LYP ${ }^{[64]}$ with the Grimme's D3 dispersion correction ${ }^{[65]}$ (B3LYP-D3) was employed along with 
the $6-31 G^{\star *}$ basis set and half of the counterpoise correction (CP). Time-dependent DFT (TDDFT) calculations were performed using the coulomb-attenuating CAMB3LYP approach ${ }^{[66]}$ with the 6-31G** basis set for the lowest-lying excited states. Excitation energies were convoluted with Gaussian functions with full-width-at-halfmaximum (FWHM) of $0.2 \mathrm{eV}$. Charge transfer was evaluated as the accumulated natural population analysis (NPA) ${ }^{[67]}$ charges on each moiety. Molecular orbitals were represented by means of the Chemcraft 1.7 software. ${ }^{[68]}$

Electrical measurements. Pressed pellets ( $P \approx 5$ US tons) were cut in rectangular shapes and contacted with silver conductive paint (RS 123-9911) and platinum wires (Goodfellow, 99.99\%, $25 \mu \mathrm{m}$ of diameter) in a 4 probe configuration (Figures S2-S3). The geometrical factors (thickness, width and length were measured using an optical microscope (width and length were determined from the top view, Figures S2-3a, and the thickness from the lateral one, Figures S2-3b). I-V curves were measured with a Keithley 6517B electrometer for ultra-high resistance/ultra-low current measurements in a two-probe configuration, i.e., applying a voltage bias between two leads and measuring the current between them. 


\section{Supporting Information}

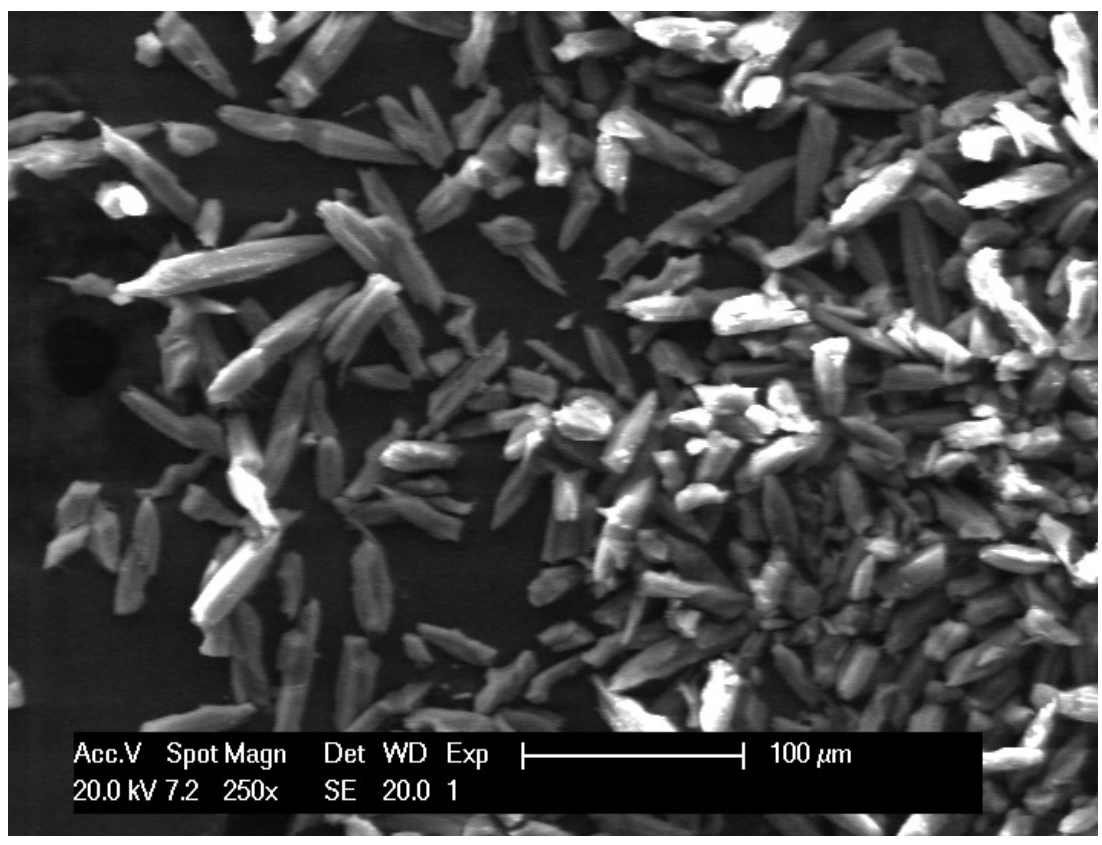

Figure S1: SEM image of $\mathbf{C}_{60} @ M U V-2$.

a

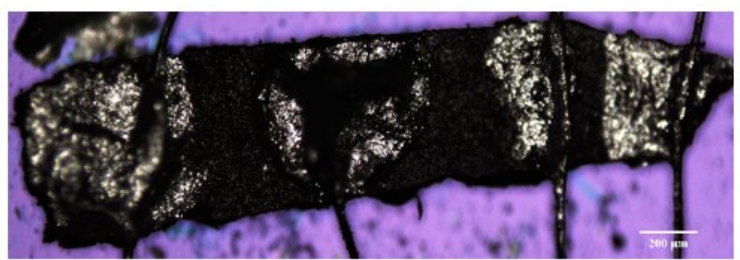

b

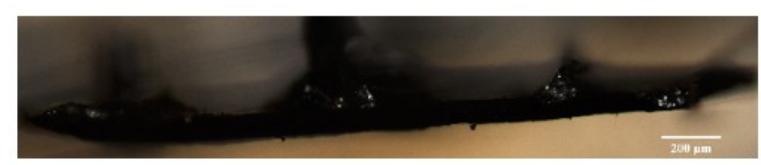

Figure S2: Top view (a) and lateral view (b) for MUV-2 electrical measurements.

a

b
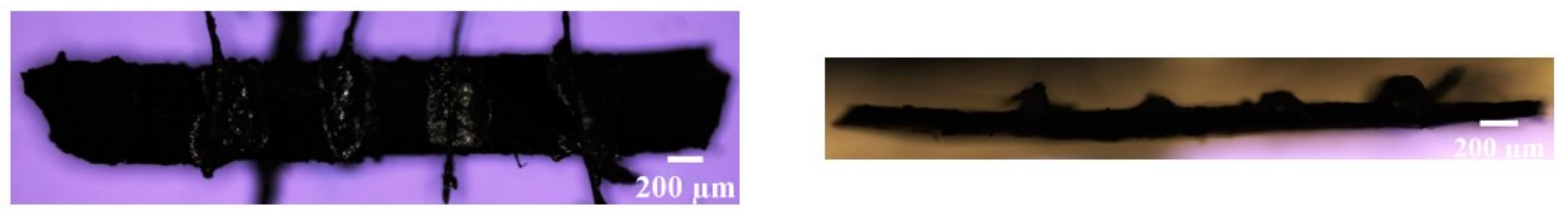

Figure S3: Top view (a) and lateral view (b) for $\mathbf{C}_{60} @$ MUV-2 electrical measurements. 
Table S1: Charge transfer (in electron charge units, e) from the electron-donor TTFTB ligand to the electron-acceptor $\mathrm{C}_{60}$ guest in the ground state (GS) and the charge-transfer $\mathrm{S}_{1}$ state (CT) of $\mathrm{C}_{60} @$ TTTFTB as a function of the distance between centroids (d). Data corresponding to the minimum-energy geometry is in bold.

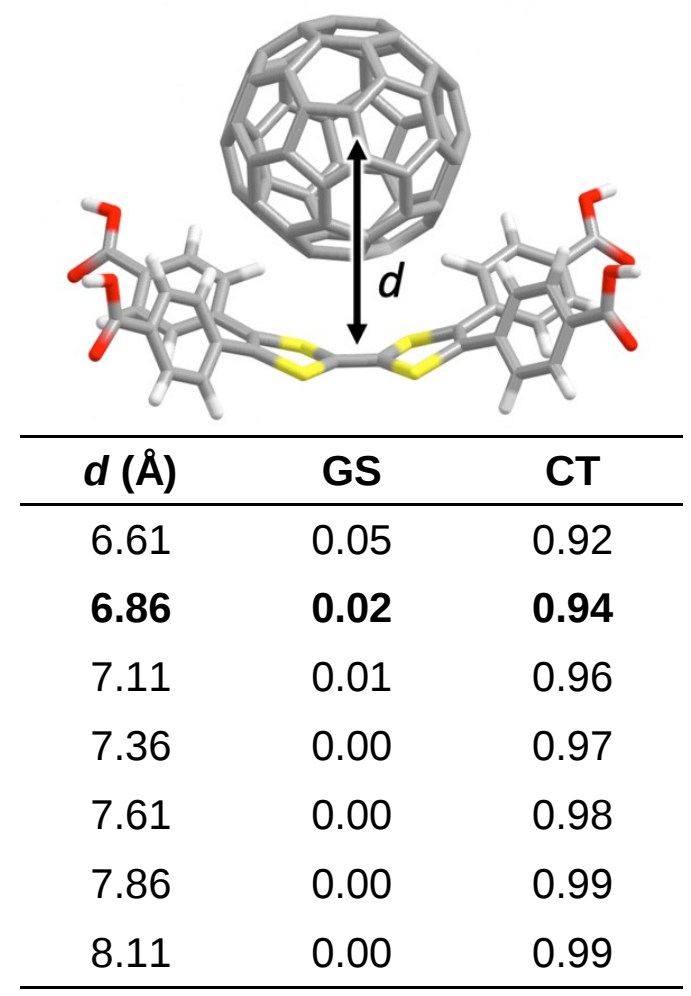


Table S2: Intense lowest-lying singlet excited states of TTFTB ligand and $\mathrm{C}_{60}$ guest, calculated at the CAM-B3LYP/6-31G** level of theory. The energy $(E)$, oscillator strength $(f)$, major monoexcitation, and description of each state are included.

\begin{tabular}{|c|c|c|c|c|c|}
\hline State & $E(\mathrm{eV})$ & $E(\mathrm{~nm})$ & $f$ & Monoexcitation & Description \\
\hline \multicolumn{6}{|c|}{ TTFTB } \\
\hline $\mathrm{S}_{1}$ & 2.907 & 426.4 & 0.0760 & $\mathrm{H} \rightarrow \mathrm{L}(68 \%)$ & $\begin{array}{c}\text { TTF } \rightarrow \\
\text { peripheral }\end{array}$ \\
\hline $\mathrm{S}_{3}$ & 3.486 & 355.7 & 0.1043 & $H \rightarrow L+4(58 \%)$ & TTF \\
\hline $\mathrm{S}_{6}$ & 4.261 & 291.0 & 0.4840 & $H \rightarrow L+3(52 \%)$ & $\begin{array}{l}\text { TTF } \rightarrow \\
\text { peripheral }\end{array}$ \\
\hline $\mathrm{S}_{8}$ & 4.430 & 279.9 & 0.5490 & $\begin{array}{c}\mathrm{H}-1 \rightarrow \mathrm{L}+1 \\
(51 \%)\end{array}$ & $\begin{array}{l}\text { TTF } \rightarrow \\
\text { peripheral }\end{array}$ \\
\hline $\mathrm{S}_{21}$ & 5.217 & 237.7 & 0.4389 & $\mathrm{H}-3 \rightarrow \mathrm{L}(15 \%)$ & $\begin{array}{l}\text { TTF } \rightarrow \\
\text { peripheral }\end{array}$ \\
\hline $\mathrm{S}_{54}$ & 6.623 & 187.2 & 0.7300 & $\begin{array}{c}\mathrm{H}-4 \rightarrow \mathrm{L}+5 \\
(13 \%) \\
\end{array}$ & TTF + peripheral \\
\hline \multicolumn{6}{|c|}{$\mathrm{C}_{60}$} \\
\hline $\mathrm{S}_{37}$ & 4.309 & 287.7 & 0.2196 & $\begin{array}{c}\mathrm{H}-2 \rightarrow \mathrm{L}+3 \\
(18 \%)\end{array}$ & - \\
\hline $\mathrm{S}_{38}$ & 4.312 & 287.6 & 0.2196 & $\begin{array}{c}\mathrm{H}-3 \rightarrow \mathrm{L}+5 \\
(21 \%)\end{array}$ & - \\
\hline $\mathrm{S}_{39}$ & 4.315 & 287.3 & 0.2206 & $\begin{array}{c}\mathrm{H}-4 \rightarrow \mathrm{L}+5 \\
(31 \%)\end{array}$ & - \\
\hline$S_{52}$ & 4.703 & 263.7 & 0.1192 & $\begin{array}{c}\mathrm{H}-6 \rightarrow \mathrm{L}+2 \\
(19 \%)\end{array}$ & - \\
\hline $\mathrm{S}_{53}$ & 4.704 & 263.6 & 0.1181 & $\begin{array}{c}\mathrm{H}-6 \rightarrow \mathrm{L}+1 \\
(21 \%)\end{array}$ & - \\
\hline $\mathrm{S}_{54}$ & 4.706 & 263.5 & 0.1133 & $\mathrm{H}-8 \rightarrow \mathrm{L}(17 \%)$ & - \\
\hline
\end{tabular}


Table S3: Evolution of $\mathrm{S}_{1} \mathrm{CT}$ excitation for $\mathrm{C}_{60} @$ TTFTB as a function of distance $d$ (as defined in Table S1) and the TTF boat dihedral angle $(\theta)$. Data corresponding to the minimum-energy geometry is in bold.

\begin{tabular}{cccc} 
& & \\
$\boldsymbol{d}(\AA)$ & $E(\mathrm{eV})$ & $\boldsymbol{E}(\mathbf{n m})$ & $\boldsymbol{f}$ \\
\hline 6.61 & 2.146 & 577.8 & 0.0284 \\
$\mathbf{6 . 8 6}$ & $\mathbf{2 . 1 4 3}$ & $\mathbf{5 7 8 . 6}$ & $\mathbf{0 . 0 1 7 8}$ \\
7.11 & 2.167 & 572.1 & 0.0103 \\
7.36 & 2.209 & 561.3 & 0.0054 \\
7.61 & 2.258 & 549.0 & 0.0027 \\
7.86 & 2.310 & 536.8 & 0.0012 \\
8.11 & 2.360 & 525.4 & 0.0005 \\
\hline $\boldsymbol{\theta}\left({ }^{\circ}\right)$ & $\boldsymbol{E}(\mathbf{e V})$ & $\boldsymbol{E}(\mathbf{n m})$ & $\boldsymbol{f}$ \\
\hline $\mathbf{1 6 2}$ & $\mathbf{2 . 1 4 3}$ & $\mathbf{5 7 8 . 6}$ & $\mathbf{0 . 0 1 7 8}$ \\
171 & 2.044 & 606.7 & 0.0131 \\
180 & 2.025 & 612.3 & 0.0079 \\
\hline
\end{tabular}


Supporting Information File 1:

File Name: $\mathbf{C}_{60} @ M U V-2$ confA

File Format: CIF

Title: Simulated structure of $\mathrm{C}_{60} @ M U V-2$ conformation A

Supporting Information File 2:

File Name: $\mathbf{C}_{60} @ M U V-2$ confB

File Format: CIF

Title: Simulated structure of $\mathrm{C}_{60} @ M U V-2$ conformation B 


\section{Acknowledgements}

This work has been supported by the European Union (ERC-2016-CoG 724681-S-CAGE) and the Spanish MICINN (CTQ2017-89528-P). G.M.E. and M.S. thank MICINN for a Ramón y Cajal and a Juan de la Cierva-Formación fellowships, respectively. J.C. acknowledges the Generalitat Valenciana for the postdoctoral APOSTD/2017/081 fellowship. Via our membership of the UK's HEC Materials Chemistry Consortium, which is funded by EPSRC (EP/L000202), this work used the ARCHER UK National Supercomputing Service (http://www.archer.ac.uk).

\section{References}

1. H. C. Zhou, J. R. Long, O. M. Yaghi, Chem. Rev., 2012, 112, 673-674.

2. G. Maurin, C. Serre, A. Cooper, G. Férey, Chem. Soc. Rev., 2017, 46, 31043107.

3. H. Furukawa, K. E. Cordova, M. O'Keeffe, O. M. Yaghi, Science, 2013, 341, 1230444.

4. A. G. Slater, A. I. Cooper, Science, 2015, 348, aaa8075.

5. S. M. J. Rogge, A. Bavykina, J. Hajek, H. Garcia, A. I. Olivos-Suarez, A. Sepúlveda-Escribano, A. Vimont, G. Clet, P. Bazin, F. Kapteijn, M. Daturi, E. V. Ramos-Fernandez, F. X. Llabrés i Xamena, V. Van Speybroeck, Chem. Soc. Rev., 2017, 46, 3134-3184.

6. L. Sun, M. G. Campbell, M. Dincă, Angew. Chem. Int. Ed., 2016, 55, 35663579 . 
7. I. Stassen, N. Burtch, A. Talin, P. Falcaro, M. Allendorf, R. Ameloot, Chem. Soc. Rev., 2017, 46, 3185-3241.

8. T. Bein, D. D. Medina, Isr. J. Chem., 2018, 4, 1089-1101.

9. M. G. Campbell, D. Sheberla, S. F. Liu, T. M. Swager, M. Dincă, Angew. Chem. Int. Ed., 2015, 54, 4349-4352.

10. G. Wu, J. Huang, Y. Zang, J. He, G. Xu, J. Am. Chem. Soc., 2017, 139, 13601363.

11. D. Sheberla, J. C. Bachman, J. S. Elias, C. Sun, Y. Shao-horn, Nat. Mat., 2017, 16, 220-224.

12. A. A. Talin, A. Centrone, A. C. Ford, M. E. Foster, V. Stavila, P. Haney, R. A. Kinney, V. Szalai, F. El Gabaly, H. P. Yoon, F. Léonard, M. D. Allendorf, Science, 2014, 343, 66-69.

13. S. K. Bhardwaj, N. Bhardwaj, R. Kaur, J. Mehta, A. L. Sharma, K. Kim, A. Deep, J. Mater. Chem. A, 2018, 6, 14992-15009.

14. C. Kung, K. Otake, C. T. Buru, S. Goswami, Y. Cui, J. T. Hupp, A. M. Spokoyny, O. K. Farha, J. Am. Chem. Soc., 2018, 140, 3871-3875.

15. D. M. D'Alessandro, Chem. Commun., 2016, 52, 8957-8971.

16. C. W. Kung, T. C. Wang, J. E. Mondloch, D. Fairen-Jimenez, D. M. Gardner, W. Bury, J. M. Klingsporn, J. C. Barnes, R. Van Duyne, J. F. Stoddart, M. R. Wasielewski, O. K. Farha, J. T. Hupp, Chem. Mater., 2013, 25, 5012-5017.

17. Q. Chen, J. Sun, P. Li, I. Hod, P. Z. Moghadam, Z. S. Kean, R. Q. Snurr, J. T. Hupp, O. K. Farha, J. F. Stoddart, J. Am. Chem. Soc., 2016, 138, 1424214245.

18. J. Calbo, M. J. Golomb, A. Walsh, J. Mater. Chem. A, 2019, DOI 10.1039/C9TA04680A.

19. A. Van Wyk, T. Smith, J. Park, P. Deria, J. Am. Chem. Soc., 2018, 140, 2756- 
2760.

20. C. Hua, P. W. Doheny, B. Ding, B. Chan, M. Yu, C. J. Kepert, D. M.

D'Alessandro, J. Am. Chem. Soc., 2018, 140, 6622-6630.

21. H. Miyasaka, Acc. Chem. Res., 2013, 46, 248-257.

22. H. W. Kroto, J. R. Heath, S. C. O'Brien, R. F. Curl, R. E. Smalley, Nature, 1985, $318,162-163$.

23. F. Giacalone, N. Martín, Chem. Rev., 2006, 106, 5136-5190.

24. N. Martín, Chem. Commun., 2006, 2093-2104.

25. A. Zieleniewska, F. Lodermeyer, A. Roth, D. M. Guldi, Chem. Soc. Rev., 2018, $47,702-714$.

26. H. Meng, C. Wang, T. Wang, Gen. Chem., 2018, 4, 180019.

27. Y. Inokuma, T. Arai, M. Fujita, Nat. Chem., 2010, 2, 780-783.

28. C. García-Simón, M. Garcia-Borràs, L. Gómez, T. Parella, S. Osuna, J.

Juanhuix, I. Imaz, D. Maspoch, M. Costas, X. Ribas, Nat. Commun., 2014, 5, 5557.

29. C. García-Simón, M. Costas, X. Ribas, Chem. Soc. Rev., 2016, 45, 40-62.

30. Y. Feng, T. Wang, Y. Li, J. Li, J. Wu, B. Wu, L. Jiang, C. Wang, J. Am. Chem. Soc., 2015, 137, 15055-15060.

31. H. Meng, C. Zhao, M. Nie, C. Wang, T. Wang, J. Phys. Chem. C, 2019, 123, 6265-6269.

32. X. Liu, M. Kozlowska, T. Okkali, D. Wagner, T. Higashino, G. Brenner-Weiß, S. M. Marschner, Z. Fu, Q. Zhang, H. Imahori, S. Bräse, W. Wenzel, C. Wöll, L. Heinke, Angew. Chem. Int. Ed., 2019, 58, 1-7.

33. S. Goswami, D. Ray, K. Otake, C. Kung, S. J. Garibay, T. Islamoglu, A. Atilgan, Y. Cui, C. J. Cramer, O. K. Farha, J. T. Hupp, Chem. Sci. 2018, 9, 4477-4482.

34. J. L. Segura, N. Martn, Angew. Chem. Int. Ed. 2001, 40, 1372-1409. 
35. N. Martín, Chem. Commun., 2013, 49, 7025-7027.

36. A. Jana, S. Bähring, M. Ishida, S. Goeb, D. Canevet, M. Sallé, J. O. Jeppesen, J. L. Sessler, Chem. Soc. Rev., 2018, 47, 5614-5645.

37. J. M. Spruell, A. Coskun, D. C. Friedman, R. S. Forgan, A. A. Sarjeant, A. Trabolsi, A. C. Fahrenbach, G. Barin, W. F. Paxton, S. K. Dey, et al., Nat. Chem., 2010, 2, 870-879.

38. M. Frasconi, T. Kikuchi, D. Cao, Y. Wu, W. G. Liu, S. M. Dyar, G. Barin, A. A. Sarjeant, C. L. Stern, R. Carmieli, C. Wang, M. R. Wasielewski, W. A. Goddard III, J. F. Stoddart, J. Am. Chem. Soc., 2014, 136, 11011-11026.

39. E. M. Pérez, L. Sánchez, G. Fernández, N. Martín, J. Am. Chem. Soc., 2006, $128,7172-7173$.

40. E. M. Pérez, M. Sierra, L. Sánchez, M. R. Torres, R. Viruela, P. M. Viruela, E. Ortí, N. Martín, Angew. Chem. Int. Ed., 2007, 46, 1847-1851.

41. E. M. Pérez, N. Martín, Chem. Soc. Rev., 2008, 37, 1512-1519.

42. D. Canevet, E. M. Pérez, N. Martín, Angew. Chem. Int. Ed. 2011, 50, 92489259.

43. S. Goeb, S. Bivaud, P. I. Dron, J. Y. Balandier, M. Chas, M. Sallé, Chem. Commun. 2012, 48, 3106-3108.

44. M. Gallego, J. Calbo, J. Aragó, R. M. Krickcalderon, F. H. Liquido, T. Iwamoto, A. K. Greene, E. A. Jackson, E. M. Pérez, E. Ortí, D. M. Guldi, L. T. Scott, N. Martín, Angew. Chem. Int. Ed., 2014, 53, 2170-2175.

45. H.-Y. Wang, L. Cui, J.-Z. Xie, C. F. Leong, D. M. D’Alessandro, J.-L. Zuo, Coord. Chem. Rev., 2017, 345, 342-361.

46. S. S. Park, E. R. Hontz, L. Sun, C. H. Hendon, A. Walsh, T. Van Voorhis, M. Dincă, J. Am. Chem. Soc., 2015, 137, 1774-1777.

47. J. Su, S. Yuan, H.-Y. Wang, L. Huang, J.-Y. Ge, E. Joseph, J. Qin, T. Cagin, J.- 
L. Zuo, H.-C. Zhou, Nat. Commun. 2017, 8, 2008.

48. H.-Y. Wang, J.-Y. Ge, C. Hua, C.-Q. Jiao, Y. Wu, C. F. Leong, D. M.

D’Alessandro, T. Liu, J.-L. Zuo, Angew. Chem. Int. Ed., 2017, 56, 5465 -5470.

49. M. Souto, A. Santiago-Portillo, M. Palomino, I. J. Vitórica-Yrezábal, B. J. C.

Vieira, J. C. Waerenborgh, S. Valencia, S. Navalón, F. Rey, H. García, G.

Mínguez Espallargas, Chem. Sci., 2018, 9, 2413-2418.

50. M. Souto, J. Romero, J. Calbo, I. J. Vitorica-Yrezabal, J. L. Zafra, J. Casado

Cordón, E. Ortí, A. Walsh, G. Mínguez Espallargas, J. Am. Chem. Soc., 2018, 140, 10562-10569.

51. M. Vicent-Morales, I. J. Vitórica-Yrezábal, M. Souto, G. Mínguez Espallargas, CrystEngComm 2019, 21, 3031-3035.

52. H. K. Chae, D. Y. Siberio-Pérez, J. Kim, Y. Go, M. Eddaoudi, A. J. Matzger, M. O'Keeffe, O. M. Yaghi, Nature, 2004, 427, 523-527.

53. H. Li, M. R. Hill, R. Huang, C. Doblin, S. Lim, A. J. Hill, R. Babarao, P. Falcaro, Chem. Commun., 2016, 52, 5973-5976.

54. V. Blum, R. Gehrke, F. Hanke, P. Havu, V. Havu, X. Ren, K. Reuter, M. Scheffler, Comput. Phys. Commun., 2009, 180, 2175-2196.

55. J. P. Perdew, A. Ruzsinszky, G. I. Csonka, O. A. Vydrov, G. E. Scuseria, L. A. Constantin, X. Zhou, K. Burke., Phys. Rev. Lett., 2008, 100, 136406.

56. A. Tkatchenko, M. Scheffler, Phys. Rev. Lett., 2009, 102, 73005.

57. J. Heyd, G. E. Scuseria, M. Ernzerhof, J. Chem. Phys., 2003, 118, 8207-8215.

58. K. T. Butler, C. H. Hendon, A. Walsh, J. Am. Chem. Soc., 2014, 136, 27032706.

59. K. Momma, F. Izumi, J. Appl. Crystallogr., 2011, 44, 1272-1276.

60. S. Keinan, J. Contreras-García, E. R. Johnson, W. Yang, P. Mori-Sánchez, A. J. Cohen, J. Am. Chem. Soc., 2010, 132, 6498-6506. 
61. J. Contreras-García, E. R. Johnson, S. Keinan, R. Chaudret, J. P. Piquemal, D. N. Beratan, W. Yang, J. Chem. Theory Comput., 2011, 7, 625-632.

62. W. Humphrey, A. Dalke, K. Schulten, J. Molec. Graph., 1996, 14, 33-38.

63. Gaussian 16, Revision A.03, M. J. Frisch, G. W. Trucks, H. B. Schlegel, G. E. Scuseria, M. A. Robb, J. R. Cheeseman, G. Scalmani, V. Barone, G. A. Petersson, H. Nakatsuji, X. Li, M. Caricato, A. V. Marenich, J. Bloino, B. G. Janesko, R. Gomperts, B. Mennucci, H. P. Hratchian, J. V. Ortiz, A. F. Izmaylov, J. L. Sonnenberg, D. Williams-Young, F. Ding, F. Lipparini, F. Egidi, J. Goings, B. Peng, A. Petrone, T. Henderson, D. Ranasinghe, V. G. Zakrzewski, J. Gao, N. Rega, G. Zheng, W. Liang, M. Hada, M. Ehara, K. Toyota, R. Fukuda, J. Hasegawa, M. Ishida, T. Nakajima, Y. Honda, O. Kitao, H. Nakai, T. Vreven, K. Throssell, J. A. Montgomery, Jr., J. E. Peralta, F. Ogliaro, M. J. Bearpark, J. J. Heyd, E. N. Brothers, K. N. Kudin, V. N. Staroverov, T. A. Keith, R. Kobayashi, J. Normand, K. Raghavachari, A. P. Rendell, J. C. Burant, S. S. Iyengar, J. Tomasi, M. Cossi, J. M. Millam, M. Klene, C. Adamo, R. Cammi, J. W. Ochterski, R. L. Martin, K. Morokuma, O. Farkas, J. B. Foresman, and D. J. Fox, Gaussian, Inc., Wallingford CT, 2016.

64. A. D. Becke, J. Chem. Phys., 1993, 98, 5648-52.

65. S. Grimme, S. Ehrlich, L. Goerigk, J. Comp. Chem., 2011, 32, 1456-1465.

66. T. Yanai, D. P. Tew, N. C. Handy, Chem. Phys. Lett. 2004, 393, 51-57.

67. F. Weinhold, J. E. Carpenter, in (Eds.: R. Naaman, Z. Vager), 1988, pp. 227236.

68. Chemcraft - graphical software for visualization of quantum chemistry computations. https://www.chemcraftprog.com 


\title{
Investigation of Charge-Transfer Interactions
}

\author{
Induced by Encapsulating Fullerene in a
}

\section{Mesoporous Tetrathiafulvalene-based Metal-Organic}

\section{Framework}

Manuel Souto*1, Joaquín Calbo², Samuel Mañas-Valero ${ }^{1}$, Aron Walsh² and Guillermo Mínguez Espallargas*1

Address:

${ }^{1}$ Instituto de Ciencia Molecular (ICMol), Universidad de Valencia, C/ Catedrático José Beltrán 2, 46980 Paterna, Spain and ²Department of Materials, Imperial College London, London SW7 2AZ, United Kingdom.

Email: Manuel Souto - manuel.souto@uv.es; Guillermo Mínguez Espallargas guillermo.minguez@uv.es

* Corresponding author

\section{Abstract}

The design of Metal-Organic Frameworks (MOFs) incorporating electroactive guest molecules in the pores has become a subject of great interest in order to install additional electrical functionalities within the framework while maintaining porosity. In this direction, understanding the charge-transfer (CT) process between the framework and the guest molecules is crucial towards the design of new electroactive MOFs. Herein, we present the encapsulation of fullerenes $\left(\mathrm{C}_{60}\right)$ in a mesoporous 
tetrathiafulvalene(TTF)-based MOF. The CT process between the electron-acceptor $\mathrm{C}_{60}$ guest and the electron-donor TTF ligand is studied in detail by means of different spectroscopic techniques and density functional theory calculations. Importantly, gas sorption measurements demonstrate that sorption capacity is maintained after encapsulation of fullerenes, whereas the electrical conductivity is increased by two orders of magnitude due to the CT interactions between $\mathrm{C}_{60}$ and the TTF-based framework.

\section{Keywords}

Metal-Organic Framework; Tetrathiafulvalene; Fullerene; Donor-Acceptor; Charge Transfer

\section{Introduction}

The emerging field of Metal-Organic Frameworks (MOFs), crystalline porous materials constructed from metallic nodes and organic linkers, has been one of the major breakthroughs in Chemistry during the last decades..$^{[1,2]}$ Because of their vast structural and functional possibilities, this class of hybrid materials has shown to find several applications in, for example, gas storage and separation, sensing or catalysis. ${ }^{[3-5]}$ In addition, electroactive MOFs combining porosity and electrical conductivity $^{[6-8]}$ have also attracted large attention during the last years in view of their potential application as chemiresistive sensors, ${ }^{[9]}$ field-effect transistors ${ }^{[10]}$ or supercapacitors ${ }^{[11]}$ among others. Whereas most of MOFs are electrical insulators, a few of such materials have shown to exhibit excellent electrical conductivity and high charge mobility based on an appropriate choice of the building units to form electrically delocalised frameworks, or by inclusion of electroactive guest molecules 
in the pores. ${ }^{[6,12-14]}$ In this direction, the incorporation of redox-active moieties ${ }^{[15-18]}$ as well as the understanding of charge-transfer (CT) processes in MOFs, ${ }^{[19-21]}$ are excellent pathways for the rational design of new electroactive frameworks exhibiting electrical conductivity and porosity at the same time.

Fullerenes $\left(\mathrm{C}_{60}\right)^{[22]}$ have demonstrated to find numerous applications in different fields, ranging from molecular electronics and nanotechnology to biomedical applications, due to their exceptional electrochemical and photophysical properties. ${ }^{[23,24]}$ In particular, understanding CT processes between electron-acceptor $\mathrm{C}_{60}$ and electron-donor molecules is fundamental for optimising photovoltaics and develop efficient solar cells. ${ }^{[25]}$ Encapsulation of $\mathrm{C}_{60}$ in $\mathrm{MOF}{ }^{[26]}$ has become a very interesting strategy for fullerene purification, ${ }^{[27-29]}$ or to incorporate additional functionalities within the MOF. ${ }^{[30-32]}$ Very recently, Farha and co-workers have demonstrated that encapsulation of $\mathrm{C}_{60}$ in a zirconium-based MOF can lead to an enhancement of electrical conductivity due to donor-acceptor (D-A) interactions between the pyrene-based ligand (donor) and fullerene (acceptor) without significant decrease of porosity. ${ }^{[33]}$

Tetrathiafulvalene (TTF) and its numerous derivatives are redox-active electron-donor molecules with unique electronic properties that have been widely used as important building units in the field of molecular electronics as conductors, switches, sensors or rectifiers. ${ }^{[34,35]}$ Several studies have also been devoted to the development of TTF-based macrocyclic systems for their use as molecular machines or for supramolecular host-guest recognition. ${ }^{[36-38]}$ In this context, D-A interactions between $\mathrm{C}_{60}$ and discrete $\pi$-extended TTF molecules have been widely studied in solution during the last years. ${ }^{[39-44]}$ In contrast, much less is known about their supramolecular interactions in solid-state polymeric structures. 
MOFs using TTF as ligands have become an interesting new class of functional porous systems since they can incorporate additional electronic properties from TTF ligands to prepare new electrically conductive and redox-active MOFs. ${ }^{[45-48]}$ Very recently, we have reported a hierarchical and highly stable TTF-based MOF, named MUV-2, which is based on the 6-connected trimeric cluster $\left[\mathrm{Fe}_{3}\left(\mu_{3} \mathrm{O}\right)(\mathrm{COO})_{6}\right]$ as secondary building unit (SBU) and tetratopic tetrathiafulvalene-tetrabenzoic (TTFTB ${ }^{4-}$ ) ligands. This MOF shows a hierarchical structure with mesoporous channels of $\sim 3 \mathrm{~nm}$ and orthogonal microporous channels of $\sim 1 \mathrm{~nm}$. In addition, it exhibits an enhanced catalytic activity for the aerobic oxidation of dibenzothiophene in diesel, ${ }^{[49]}$ and a reversible continuous breathing upon adsorption of different solvents. ${ }^{[50]}$ Importantly, the planarity of the TTF ligands can be modulated by the breathing behaviour, which directly impacts on its electrochemical properties. ${ }^{[50,51]}$ In view of the remarkable electron-donor character of the TTF-based ligands, herein we present the encapsulation of $\mathbf{C}_{60}$ in MUV-2 ( $\left.\mathbf{C}_{60} @ \mathbf{M U V - 2}\right)$ (Figure 1). A detailed study on the CT interactions between the electron-donor TTF ligands from the framework and the electron-acceptor fullerenes has been carried out by different spectroscopic techniques and theoretical calculations. Gas sorption measurements demonstrate that permanent porosity is retained, whereas electrical measurements show that conductivity is enhanced after $\mathrm{C}_{60}$ encapsulation. 


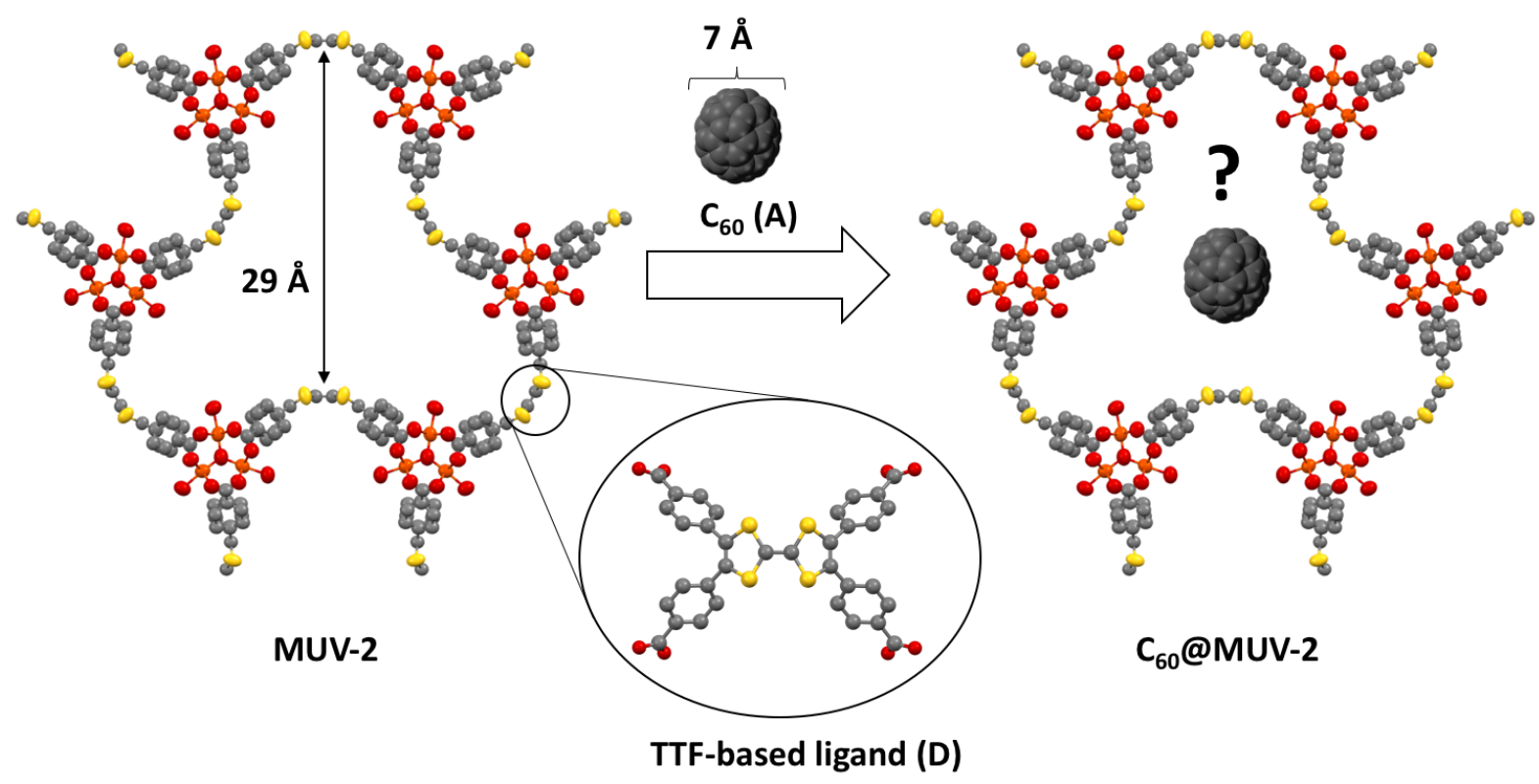

Figure 1: Schematic representation of the encapsulation of the electron-acceptor (A) $\mathrm{C}_{60}$ in the electron-donor (D) TTF-based MUV-2. Grey, yellow, orange and red spheres represent $\mathrm{C}, \mathrm{S}, \mathrm{Fe}$ and $\mathrm{O}$ atoms, respectively.

\section{Results and Discussion}

\section{Synthesis and characterisation of $\mathrm{C}_{60} @ M U V-2$}

\section{Synthesis and encapsulation of $\mathrm{C}_{60}$ into MUV-2}

MUV-2 was synthesised as previously described based on the solvothermal reaction of tetrathiafulvalene tetrabenzoic acid $\left(\mathrm{H}_{4}\right.$ TTFTB), the preformed cluster $\left[\mathrm{Fe}_{3} \mathrm{O}\left(\mathrm{CH}_{3} \mathrm{COO}\right)_{6}\right] \mathrm{ClO}_{4}$ and acetic acid as modulator, in dimethylformamide (DMF). ${ }^{[49]}$ In order to activate the material, MUV-2 was exhaustively washed with DMF, methanol and heated at $150 \stackrel{\circ}{\circ}$ for 2 hours. Encapsulation of $\mathrm{C}_{60}$ was achieved adapting a reported procedure ${ }^{[33]}$ by immersing the activated microcrystalline powder of MUV-2 in a saturated solution of $\mathrm{C}_{60}$ in o-dichlorobenzene for three days at $60{ }^{\circ} \mathrm{C}$. Then, the material was washed with o-dichlorobenzene in order to remove the physisorbed $\mathrm{C}_{60}$ on the MOF surface, washed with methanol and dried at $150{ }^{\circ} \mathrm{C}$ for 
2 hours. Powder X-ray diffraction (PXRD) pattern of $\mathbf{C}_{60} @ M$ MU-2 shows that the principal peak remains at $3.4^{\circ}$ confirming that crystallinity is maintained after encapsulation of $\mathrm{C}_{60}$ and removal of the solvent (Figure 2). The needle-like morphology of $\mathbf{C}_{60} @$ MUV-2 also remained similar to the one of MUV-2 as confirmed by Scanning Electron Microscopy (SEM) (Figure S1).

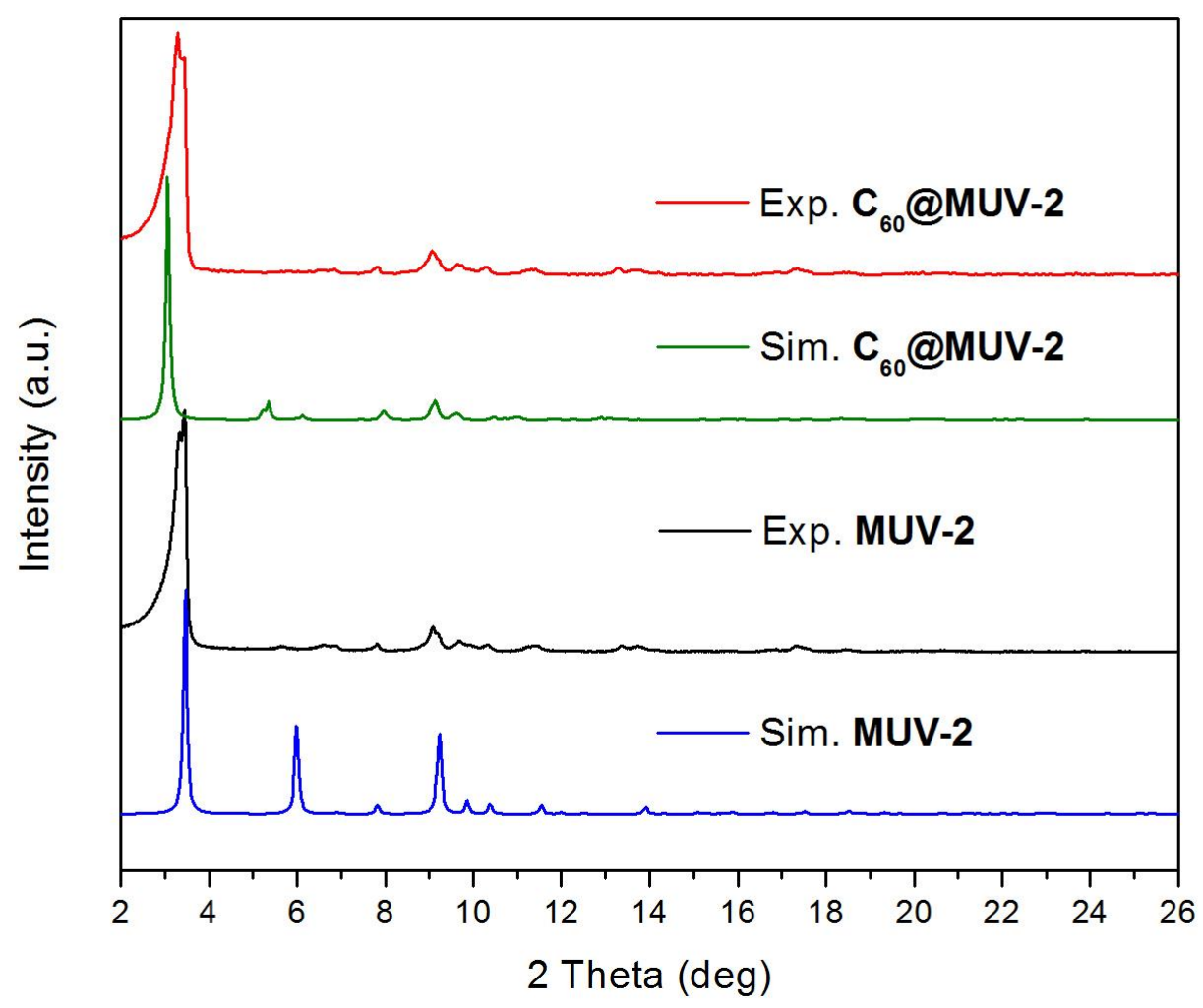

Figure 2: Powder X-ray diffraction (PXRD) patterns of simulated and experimental desolvated MUV-2 and $\mathbf{C}_{60} @ M U V-2$.

\section{Raman and UV-vis spectroscopy}

Raman spectra of $\mathbf{C}_{60}, \mathbf{M U V}-2$ and $\mathbf{C}_{60} @ \mathbf{M U V - 2}$ crystals were measured using a Raman laser of $785 \mathrm{~nm}$ (Figure 3a). The presence of Raman bands at 218, 284 and $490 \mathrm{~cm}^{-1}$ evidences the encapsulation of $\mathrm{C}_{60}$ in MUV-2, whereas the broadening and shift of the bands towards larger frequencies are indicative of the charge-transfer 
interaction between the electron-acceptor $\mathrm{C}_{60}$ and the electron-donor TTF ligands of the framework. ${ }^{[3,52]}$ On the other hand, the UV-vis spectrum of $\mathbf{C}_{60} @ \mathbf{M U V - 2}$ crystals dispersed in $\mathrm{KBr}$ pellets (Figure $3 \mathrm{~b}$ ) shows the presence of two new bands around 260 and $350 \mathrm{~nm}$, which can be assigned to $\mathrm{C}_{60}$, whereas a broad band from 450 to $800 \mathrm{~nm}$ can be designated to an intermolecular charge-transfer (CT) excitation between the $\mathrm{C}_{60}$ and TTF ligands, as supported by theoretical calculations (see below). The energy range for the CT band is in agreement with the HOMO-LUMO gap calculated from electrochemical experiments, as the redox potential of TTF linkers can be tuned from 1.1 to $2.2 \mathrm{~V}(\mathrm{vs} . \mathrm{Ag} / \mathrm{AgCl})^{[50]}$ depending on the planarity of the ligands and the redox potential of $\mathrm{C}_{60}$ is $-0.33 \mathrm{~V}(\mathrm{vs} . \mathrm{Ag} / \mathrm{AgCl})$, thus the $\mathrm{CT}$ band should appear between 1.4-2.5 eV (495-885 nm).
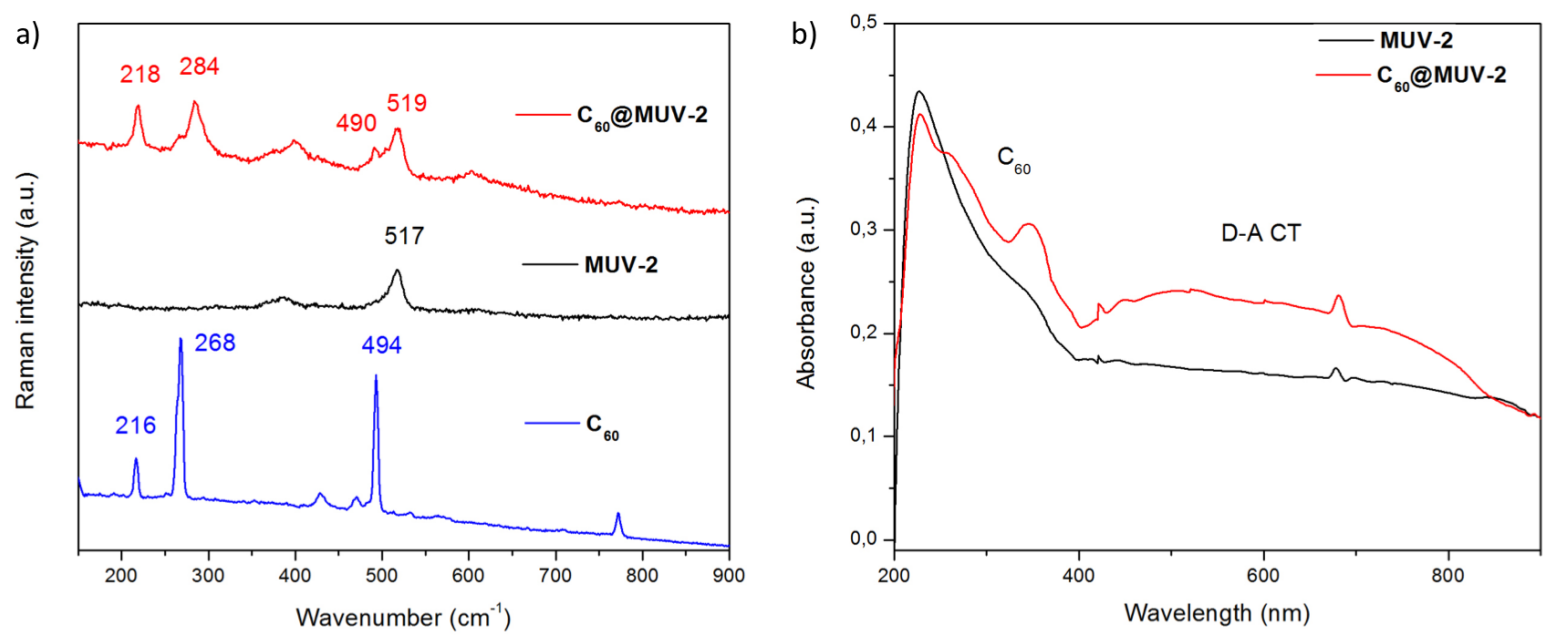

Figure 3: a) Raman spectra of $\mathbf{C}_{60}, \mathbf{M U V - 2}$ and $\mathbf{C}_{60} @ \mathbf{M U V - 2 . ~ b ) ~ S o l i d - s t a t e ~ U V - v i s ~}$ spectra of MUV-2 and $\mathbf{C}_{60} @ \mathbf{M U V - 2}$. The spectra were recorded by dispersing the samples in $\mathrm{KBr}$ pellets.

\section{Gas sorption measurements}

The porosity of $\mathbf{C}_{60} @ M U V-2$ was studied by means of $\mathrm{N}_{2}$ and $\mathrm{CO}_{2}$ isotherms measurements (Figure 4). Nitrogen adsorption isotherm at $77 \mathrm{~K}$ showed a combination of Types I and IV isotherms (Figure 4a), as in the case of MUV-2, 
indicating the presence of micropores and mesopores in the framework. $\mathbf{C}_{60} @ \mathbf{M U V - 2}$ has a Brunauer-Emmett-Teller (BET) surface area of $1040 \mathrm{~m}^{2} / \mathrm{g}$, which is slightly lower than that of MUV-2 $\left(1190 \mathrm{~m}^{2} / \mathrm{g}\right)$, thus confirming that porosity is retained after encapsulation of $\mathrm{C}_{60}$, in agreement with other reported examples. ${ }^{[3,53]}$ The pore volume decreased from $0.53 \mathrm{~cm}^{3} / \mathrm{g}$ to $0.44 \mathrm{~cm}^{3} / \mathrm{g}$ after encapsulation of $\mathrm{C}_{60}$ in MUV2, whereas the average pore diameter calculated by means of the Barret-JoynerHalenda (BJH) method was found to be similar in both cases $(\sim 35 \AA)$. The quantity of fullerene encapsulated in MUV-2 was estimated from the decrease in pore volume, obtaining a value of around $0.7 \mathrm{C}_{60}$ per 3 TTF ligands, almost 1 fullerene per section of the void. This low encapsulation can be explained by diffusion issues or by weak interactions between the $\mathrm{C}_{60}$ and the framework, which are not strong enough to keep the $\mathrm{C}_{60}$ retained during the washing procedure. On the other hand, $\mathrm{CO}_{2}$ isotherm on $\mathbf{C}_{60} @ M U V-2$ at $298 \mathrm{~K}$ also showed a small decrease in the gas sorption capacity (Figure 4b), especially at high pressures $(7.7$ and $5.3 \mathrm{mmol} \mathrm{CO} / \mathrm{g}$ at $18 \mathrm{bar}$ for MUV-2 and $\mathbf{C}_{60} @ M U V-2$, respectively).
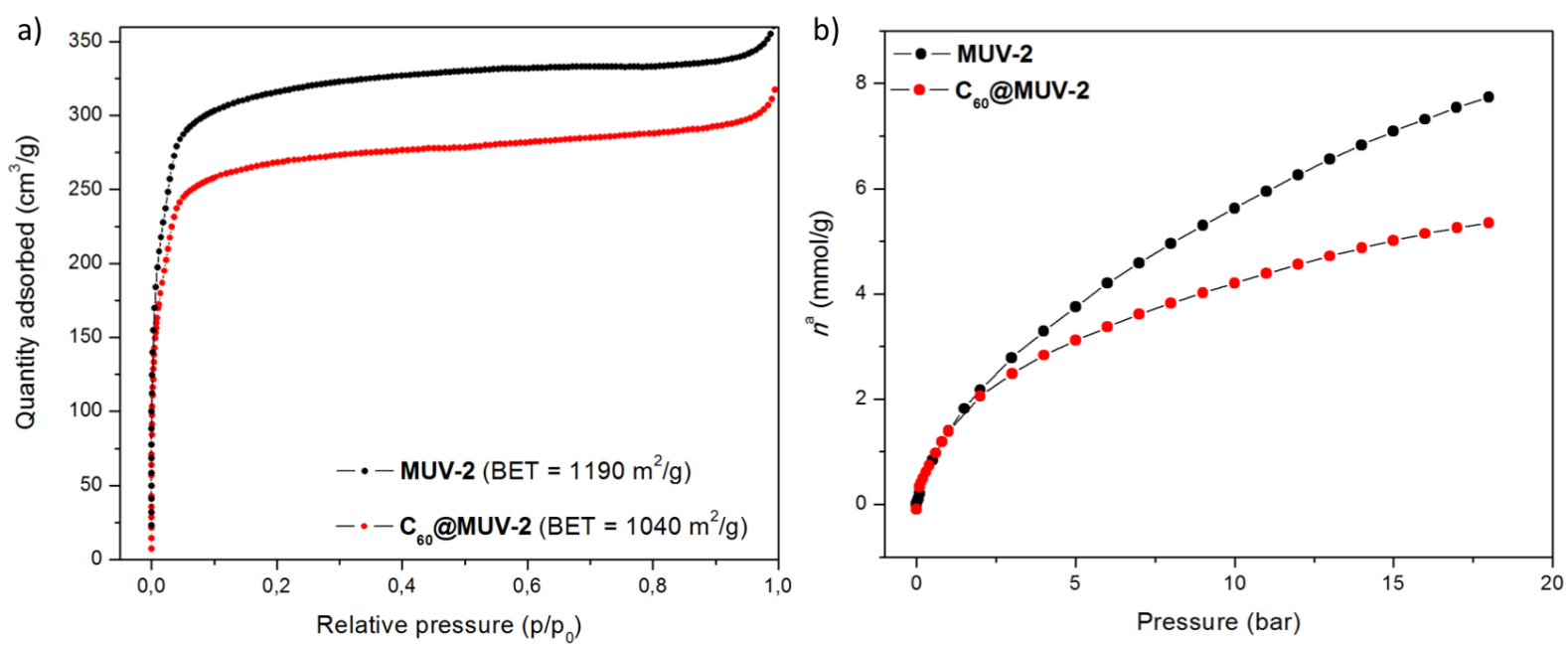

Figure 4: a) Nitrogen adsorption isotherms at $77 \mathrm{~K}$ and b) high-pressure $\mathrm{CO}_{2}$ adsorption isotherms at $298 \mathrm{~K}$, on MUV-2 (black) and $\mathbf{C}_{60} @$ MUV-2 (red). 


\section{Theoretical calculations}

In order to get further insight into the donor-acceptor interactions between $\mathbf{C}_{60}$ and the TTF-based MOF, theoretical calculations were performed under the density functional theory (DFT). The MUV-2 framework was modelled as previously described, ${ }^{[50]}$ with a high-spin $\mathrm{Fe}(\mathrm{III})$ configuration and one fullerene $\mathrm{C}_{60}$ guest molecule per pore (according to the experimental encapsulation efficiency). The hostguest $\mathbf{C}_{60} @ M U V-2$ system was fully optimized under periodic boundary conditions using the PBEsol functional with dispersion corrections (see the Experimental section for details). We initially modelled the fullerene $\mathrm{C}_{60}$ guest in the middle of the MUV-2 mesopore. After several relaxation steps, the $\mathrm{C}_{60}$ was able to accommodate in one of the three cavities to interact favourably with the TTF-based ligand. We explored two possible conformations for the host-guest $\mathbf{C}_{60} @$ MUV-2 system (A and B; Figure 5a). In conformer A, the fullerene ball remains in the void between two TTFTB ligands, approaching one of them with short $\mathrm{C}\left(\mathrm{C}_{60}\right) \cdots \mathrm{S}$ (TTFTB) and $\mathrm{C}\left(\mathrm{C}_{60}\right) \cdots$ benzene $($ TTFTB) contacts calculated at 3.4 and $3.5 \AA$, respectively (Figure 5b). In conformer B, fullerene remains over the TTFTB ligand, promoting an efficient concave-convex complementarity with a large amount of noncovalent interactions

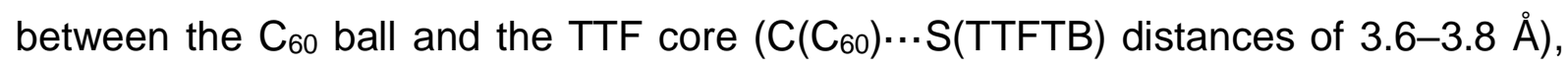
and stabilizing $\mathrm{CH} \cdots \pi$ contacts between the benzene rings of TTFTB and the fullerene $(2.5 \AA$, Figure 5). Analysis of the $\mathrm{NCl}$ index allows visualization of the noncovalent interactions between the TTFTB ligand and the $\mathrm{C}_{60}$ guest, showing a significantly larger $\mathrm{NCl}$ surface for conformer $\mathrm{B}$ compared to $\mathrm{A}$ (Figure $5 \mathrm{~b}$ ). 
a)
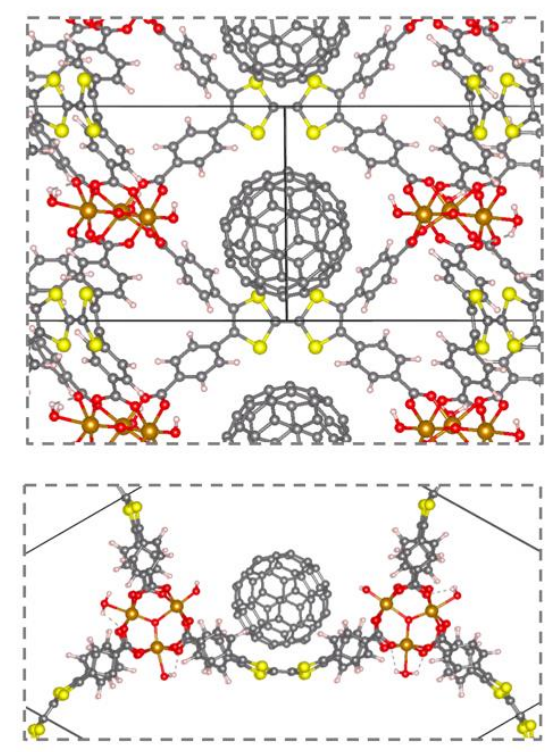

b)

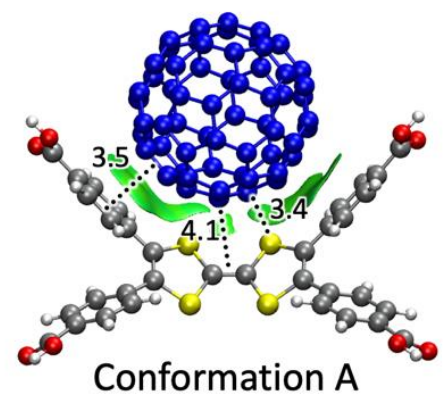

Conformation B
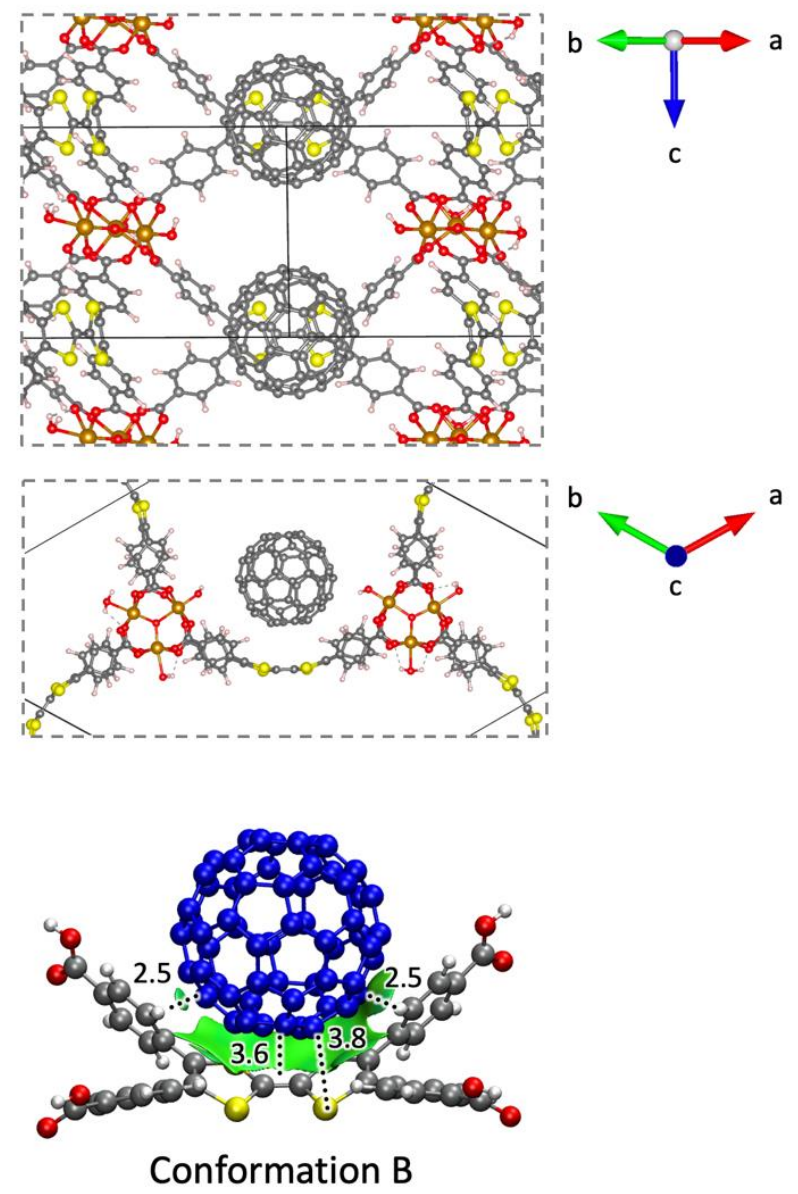

Figure 5: a) Minimum-energy crystal structure calculated for conformations $A$ and $B$ of host-guest $\mathbf{C}_{60} @ M U V-2$ at the PBEsol level in periodic boundary conditions (PBC). b) Supramolecular assemblies of $\mathbf{C}_{60} @$ TTFTB extracted from the PBC-optimized $\mathbf{C}_{60} @$ MUV-2 system in arrangements A and B. Intermolecular short contacts (in $\AA$ ) and $\mathrm{NCl}$ surfaces (reduced density gradient isovalue $=0.3$ a.u.) are displayed. Interaction energies $\left(E_{\mathrm{int}}\right)$ of -20.01 and $-23.74 \mathrm{kcal} / \mathrm{mol}$ are calculated for $\mathrm{C}_{60} @$ TTFTB in conformer $\mathrm{A}$ and $\mathrm{B}$, respectively. Fullerene $\mathrm{C}_{60}$ is coloured in blue for better viewing.

Accurate hybrid DFT molecular calculations including dispersion corrections were performed to quantify the total stabilization gained when $\mathrm{C}_{60}$ interacts with MUV-2 in arrangements $\mathrm{A}$ and $\mathrm{B}$. Interaction energies $\left(E_{\text {int }}\right)$ were calculated for the cluster 
C60@TTFTB at the B3LYP-D3/6-31G** level of theory with counterpoise correction, using the minimum-energy geometry previously obtained under periodic boundary conditions (see Experimental section). Theoretical calculations indicate that $\mathrm{C}_{60}$ favourably interacts with the TTFTB ligand, with large $E_{\text {int }}<-20 \mathrm{kcal} / \mathrm{mol}$ in both arrangements. Conformer B, in which the fullerene is placed over the TTF moiety promoting an efficient concave-convex complementarity (Figure 5), is predicted the most stable arrangement, with an $E_{\text {int }}$ of $-23.74 \mathrm{kcal} / \mathrm{mol}$ (ca. $4 \mathrm{kcal} / \mathrm{mol}$ more stable than conformer A). Henceforth, we focus subsequent analysis on conformation B.

Electronic structure calculations indicate that $\mathbf{C}_{60} @ \mathbf{M U V - 2}$ presents a small bandgap calculated to be $0.90 \mathrm{eV}$ in spin-up or $\alpha$-channel, and $0.72 \mathrm{eV}$ in spin-down or $\beta$ channel (Figure 6) - slightly smaller than that predicted for pristine MUV-2 $(0.86 \mathrm{eV}$ in $\beta$-channel). ${ }^{[50]}$ Analysis of the projected density of states (PDoS) indicates that the valence band maximum (VBM) in $\mathbf{C}_{60} @ M U V-2$ corresponds to the electron-rich TTF unit (Figure 6a). The highest-occupied crystal orbital (HOCO) displays the typical shape of the TTF HOMO and confirms the TTF-nature of the VBM (Figure 6b). In the $\alpha$-channel, the conduction band minimum (CBM) is described by the fullerene moiety, being the lowest-unoccupied crystal orbital (LUCO) completely localized on the $\mathrm{C}_{60}$ ball. Otherwise, the CBM in the $\beta$-channel is best described by the unoccupied Fe $d$ orbitals of the inorganic cluster of the MOF, the eigenstates corresponding to the fullerene being only $0.2 \mathrm{eV}$ above in energy (Figure 6). Due to the relatively low bandgap, the nature of the frontier crystal orbitals and the close proximity between the electroactive donor TTF and acceptor $\mathrm{C}_{60}$ moieties, charge-transfer processes are expected upon light irradiation. 
a)

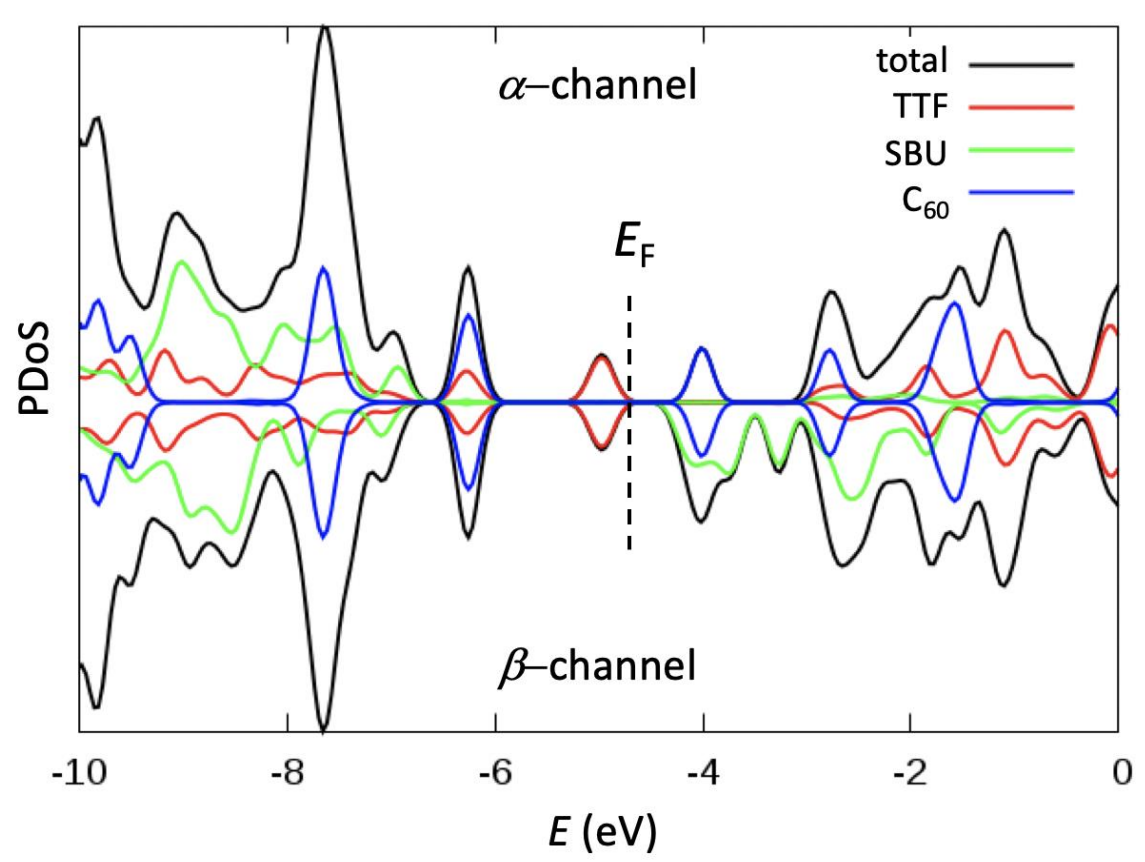

b)
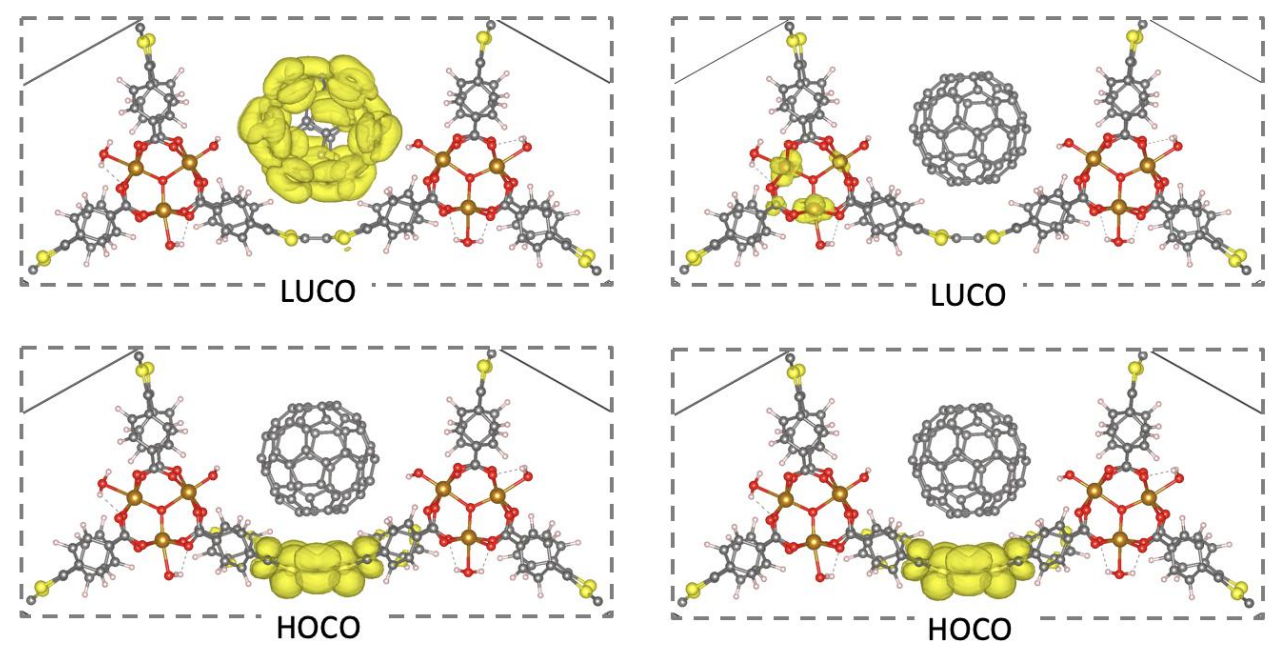

$\alpha$-channel

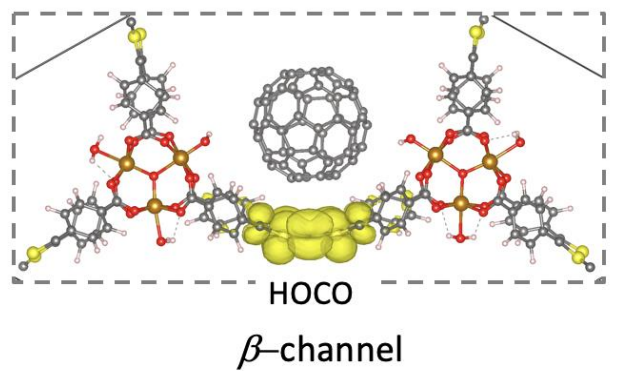

Figure 6: a) Projected density of states (PDoS) for the host-guest $\mathbf{C}_{60} @ \mathbf{M U V - 2}$ system, with contributions from the TTF core, the secondary building unit (SBU), and the fullerene $\mathrm{C}_{60}$. The Fermi level $\left(E_{\mathrm{F}}\right)$ is indicated, and the energy reference is set to vacuum. b) Highest-occupied (HOCO) and lowest-unoccupied (LUCO) crystal orbitals in the two spin channels for $\mathbf{C}_{60} @ \mathbf{M U V - 2 . ~}$

Donor-acceptor interactions in $\mathbf{C}_{60} @ \mathbf{M U V - 2}$ were first assessed at the ground state electronic configuration. The electron density difference between the framework 
interacting with $\mathrm{C}_{60}\left(\mathbf{C}_{60} @ \mathbf{M U V}-2\right)$ and the individual moieties $\left(\mathbf{C}_{60}+\mathbf{M U V - 2}\right)$ suggests a partial charge transfer from the TTF to the fullerene ball. Blue regions in Figure 7 indicate that the electron density is depleted from the TTF unit, especially from the $S$ lone pairs, and is accumulated (yellow volumes) in the fullerene regions close to the TTFTB ligand. The partial charge transfer from the donor TTFTB ligand to the acceptor fullerene moiety in the ground state is calculated to be as small as $0.02 e$, with an exponential decay as a function of the $\mathrm{C}_{60} \cdots \mathrm{TTF}$ intermolecular distance (Table S1).

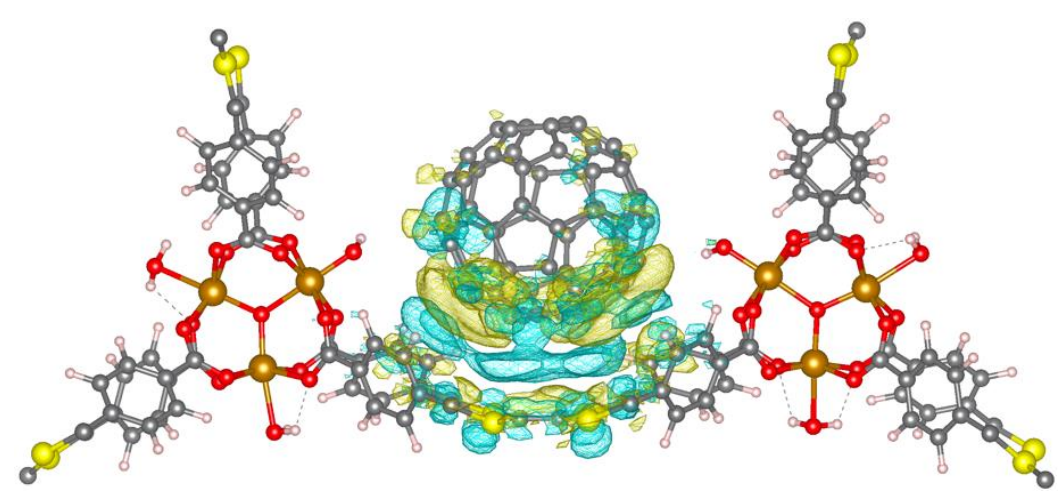

Figure 7: Electron density difference between host-guest $\mathbf{C}_{60} @ \mathbf{M U V - 2}$ and the constituting moieties $\left(\mathbf{C}_{60}+\mathbf{M U V - 2}\right)$. Blue and yellow regions indicate charge depletion and accumulation, respectively.

Time-dependent DFT molecular calculations were performed at the CAM-B3LYP/6$31 \mathrm{G}^{* *}$ level (see the Experimental section) to shed light into the photoinduced charge-transfer process of $\mathbf{C}_{60} @ \mathbf{M U V - 2}$. Figure 8 displays the simulated absorption spectra predicted for the cluster $\mathbf{C}_{60} @$ TTFTB system in the most stable arrangement $\mathrm{B}$, the TTFTB ligand, and the fullerene guest (the triplet excitation energies are indicated). The high-energy region (<400 nm) of the experimental UV-vis absorption spectrum of $\mathbf{C}_{60} @ M U V-2$ is dominated by the MUV-2 framework (Figure 3b). Theoretical calculations predict several intense transitions in the $<300 \mathrm{~nm}$ region for 
TTFTB ligand (Table S2) that explain the experimental wide band with maximum at ca. 230 nm recorded for MUV-2 and $\mathbf{C}_{60} @$ MUV-2. These transitions are described by $\pi-\pi^{*}$ electronic promotions involving the TTF and the peripheral carboxybenzene groups $\left(\mathrm{S}_{6}, \mathrm{~S}_{8}, \mathrm{~S}_{21} \mathrm{~S}_{54}\right)$ in the TTFTB ligand (Table $\left.\mathrm{S} 2\right)$. Singlet excited states $\mathrm{S}_{1}$ and $\mathrm{S}_{3}$ are predicted with less intensity (oscillator strength $f<0.2$ ), are described by TTF $\rightarrow$ benzene and TTF-centred monoexcitations, respectively, and give rise to the shoulder experimentally recorded at ca. 350 nm for $\mathbf{C}_{60} @ M U V-2$ (Table S2). On the other hand, the predicted singlet excited states of fullerene $S_{37}-S_{39}(f \approx 0.2)$ and $S_{52}-$ $\mathrm{S}_{54}(f \approx 0.1)$ in the region of 280 and $260 \mathrm{~nm}$ (Table S2), respectively, correlate with the experimental features that appear at $325 \mathrm{~nm}$ and $275 \mathrm{~nm}$ in host-guest $\mathbf{C}_{60} @ M U V-2$ (Figure 3b).

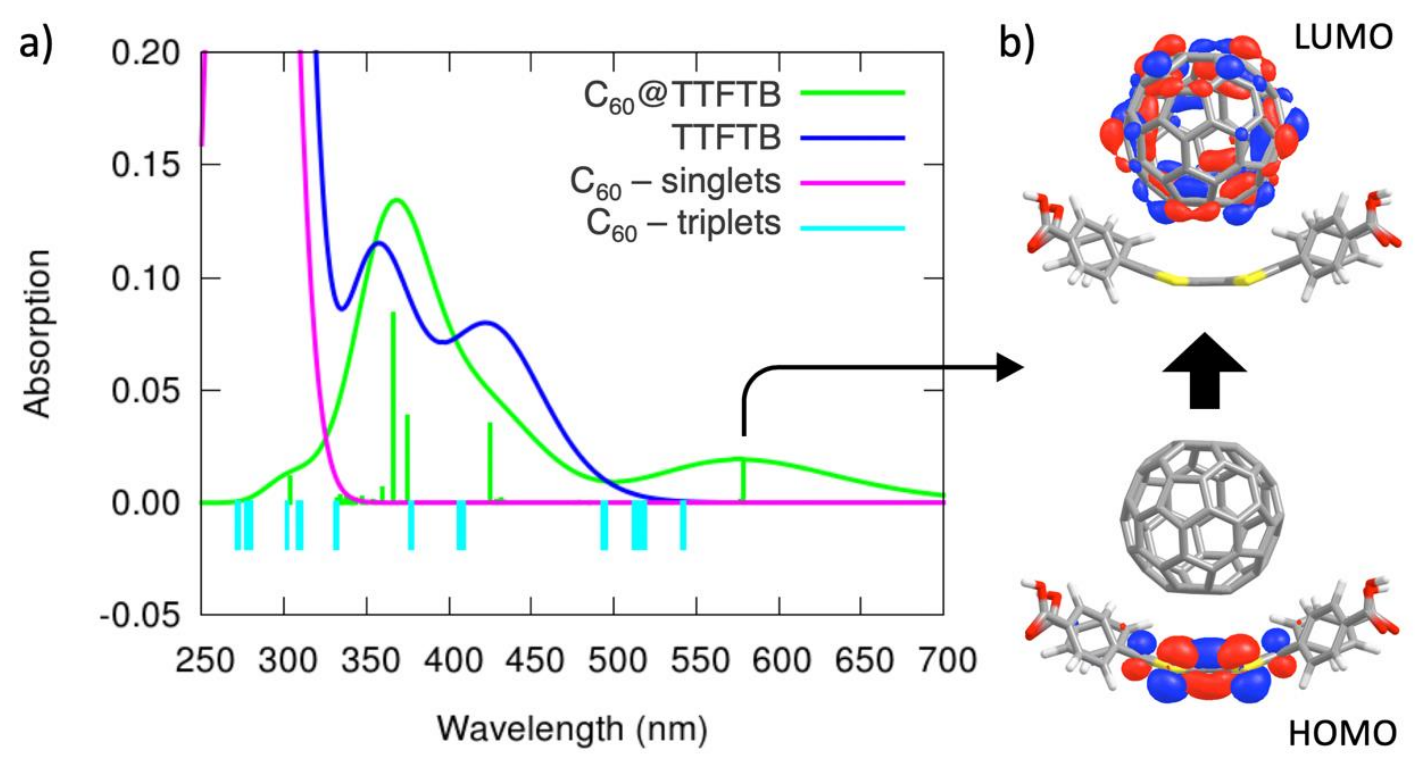

Figure 8: a) TDDFT absorption spectra calculated for host-guest $\mathbf{C}_{60} @$ TTFTB (including vertical excitation energies), TTFTB ligand, and fullerene $\mathrm{C}_{60}$ (singlets and triplet energies). b) Monoelectronic excitation that describes the nature of the lowestlying singlet excited state $\mathrm{S}_{1}$ of $\mathbf{C}_{60} @ T T F T B$. 
Importantly, a new singlet excitation is computed for $\mathbf{C}_{60} @$ TTFTB $\left(S_{1}\right)$ at 578 nm, which is not predicted either for the TTFTB ligand or the fullerene $\mathrm{C}_{60}$ (Figure 8). This transition has relatively small intensity $(f=0.018)$, and can be described by oneelectron promotion from the TTF to the $\mathrm{C}_{60}$, i.e. it has a charge-transfer (CT) nature (Figure $8 \mathrm{~b})$. The position of this CT excitation $(578 \mathrm{~nm}$ ) nicely agrees with the lowenergy broad band that appears in the experimental absorption spectrum of $\mathbf{C}_{60} @ M U V-2$, centred at $550 \mathrm{~nm}$ and expanding up to $800 \mathrm{~nm}$. In fact, theoretical calculations indicate that the intensity and energy of the $S_{1}$ CT transition in $\mathrm{C}_{60} @$ TTFTB is significantly affected by the intermolecular TTF... $\mathrm{C}_{60}$ distance and the characteristic TTF boat dihedral angle (Table S3). The charge transfer from the TTF to the fullerene ball in the $S_{1} C T$ excitation of $C_{60} @$ TTFTB is calculated to be of nearly 1 electron (0.94e at the minimum-energy geometry, Table S1).

\section{Electrical measurements}

In order to analyse the possible enhancement of electrical conductivity after encapsulation of $\mathrm{C}_{60}$ in MUV-2, transport measurements for MUV-2 and $\mathbf{C}_{60} @ \mathbf{M U V - 2}$ were performed using two-contact probe pressed-pellet devices measured at room temperature $(300 \mathrm{~K})$ (Figure 9). Interestingly, pellet of $\mathbf{C}_{60} @ \mathbf{M U V}-2$ shows an increase of around two orders of magnitude $\left(\sigma=4.7 \cdot 10^{-9} \mathrm{~S} / \mathrm{cm}\right)$ compared to the very resistive MUV-2 $\left(\sigma=3.7 \cdot 10^{-11} \mathrm{~S} / \mathrm{cm}\right.$, Table 1$)$. This enhancement of the electrical conductivity can be explained by the D-A charge transfer from the TTF linkers to the $\mathrm{C}_{60}$ since the fullerene is acting as a dopant introducing charge carriers within the framework. However, this enhancement in conductivity is lower in comparison to other reported systems ${ }^{[14,32]}$ probably due to the low ratio between $\mathrm{C}_{60}$ and TTF (1:4) and the long distances between the TTF moieties $(9.6 \AA$ along the $c$ axis), which could prevent the charge delocalisation along the framework. 


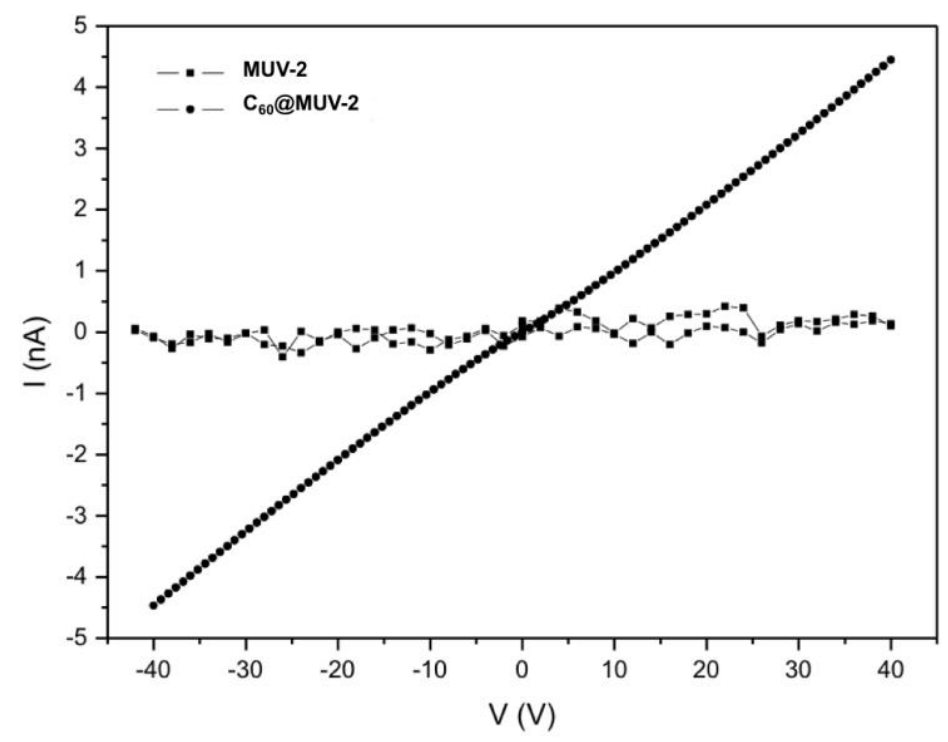

Figure 9: Current (I) - Voltage (V) plot for pressed pellets of MUV-2 and $\mathrm{C}_{60} @ M U V-2$ at $300 \mathrm{~K}$.

Table 1: Geometrical factors (length I, width w, and thickness t), resistance obtained by the linear fit of the ohmic regime of the I-V curves and conductivity for MUV-2 and $\mathrm{C}_{60} @ M U V-2$, at $300 \mathrm{~K}$.

\begin{tabular}{|c|c|c|c|c|c|}
\hline Sample & $\mathrm{I}(\mu \mathrm{m})$ & $\mathbf{w}(\mu \mathrm{m})$ & $\mathbf{t}(\mu \mathrm{m})$ & $\mathbf{R}(\boldsymbol{\Omega})$ at $\mathbf{3 0 0} \mathbf{K}$ & $\sigma(\mathbf{S} / \mathbf{c m})$ at 300 K \\
\hline MUV-2 & 110 & 480 & 65 & $(9.5 \pm 0.6) \cdot 10^{11}$ & $3.7 \cdot 10^{-11}$ \\
\hline $\mathbf{C}_{60} @ M U V-2$ & 325 & 540 & 150 & $(8.510 \pm 0.015) \cdot 10^{9}$ & $4.7 \cdot 10^{-9}$ \\
\hline
\end{tabular}

\section{Conclusion}

In summary, we have reported the encapsulation of $\mathrm{C}_{60}$ in a mesoporous TTF-based MOF (MUV-2). Charge-transfer interactions between $\mathrm{C}_{60}$ and TTF ligands from the framework in $\mathbf{C}_{60} @ M U V-2$ were confirmed by different spectroscopic techniques and theoretical calculations. Interestingly, after encapsulation of fullerene, gas sorption measurements demonstrated that mesoporosity of the MOF is maintained, and electrical measurements revealed an increase of around 2 orders of magnitude in conductivity, which can be explained by charge-transfer donor-acceptor (TTF $\rightarrow \mathrm{C}_{60}$ ) 
interactions. Current research is focused on the improvement of electrical conductivity in MUV-2 and the photophysical characterisation of the charge transfer in $\mathbf{C}_{60} @ M U V-2$.

\section{Experimental}

General methods and materials. All reagents and solvents employed for the syntheses were of high purity grade and were purchased from Sigma-Aldrich Co., and TCl. Powder X-ray diffraction spectra were recorded using $0.7 \mathrm{~mm}$ borosilicate capillaries that were aligned on an Empyrean PANalytical powder diffractometer, using $\mathrm{Cu} \mathrm{K} \alpha$ radiation $(\lambda=1.54056 \AA$ ). Raman spectra were acquired with a microRaman (model XploRA ONE from Horiba, Kyoto, Japan) with a grating of 1200 $\mathrm{gr} / \mathrm{mm}$ and a wavelength of $785 \mathrm{~nm}$. UV-vis absorption spectra were recorded on a Jasco V-670 spectrophotometer in baseline mode from 400 to $800 \mathrm{~nm}$ range. The absorption spectra were measured on the solid state by dispersed the crystals on $\mathrm{KBr}$ pellets. Nitrogen isotherms were measured using a TriStar II PLUS apparatus (Micromeritics) at $77 \mathrm{~K}$. The BET surface area was calculated by using the BrunauerEmmett-Teller equation. The high-pressure $\mathrm{CO}_{2}$ adsorption isotherms were measured in a IGA-100 gravimetric sorption analyzer (Hiden Isochema) and the sample was degassed for 2 hours at $150 \stackrel{\circ}{ } \mathrm{C}$ under vacuum.

Synthesis of MUV-2 and encapsulation of $\mathrm{C}_{60}$. MUV-2 was synthesised and characterised as previously reported. ${ }^{[49]}$ Then $30 \mathrm{mg}$ of fullerene $\left(\mathrm{C}_{60}\right)$ was dissolved in $2 \mathrm{~mL}$ of $o$-dichlorobenzene and activated MUV-2 $(10 \mathrm{mg})$ was added to it. The vial was heated at $60{ }^{\circ} \mathrm{C}$ for 3 days and the MOF was then exhaustively washed with 0 - 
dichlorobenzene to remove any physisorbed $\mathrm{C}_{60}$ on the MOF surface, washed with methanol and finally dried at $150{ }^{\circ} \mathrm{C}$ for 2 hours.

Computational Details. Theoretical calculations were performed under the density functional theory framework. Periodic boundary conditions (PBC) calculations were carried out with the FHI-AIMS (Version 171221) software. ${ }^{[54]}$ MUV-2 was modelled as previously described, with $\mathrm{Fe}(\mathrm{III})$ ions in a high-spin $d^{5}$ configuration. Guest $\mathrm{C}_{60}$ molecule was rationally inserted into the bigger mesoporous channel of MUV-2 in the most plausible sites, and the geometry of the host-guest $\mathbf{C}_{60} @ \mathbf{M U V}-\mathbf{2}$ system was fully relaxed at the PBEsol functional ${ }^{[55]}$ with tier 1 basis set. Dispersion corrections were added according to the Hirshfeld partitioning of the electron density (Tkatchenko-Scheffler method). ${ }^{[56]}$ Electronic structure calculations were performed for band structure analysis using the hybrid HSE06 functional[ ${ }^{[57]}$ and tier 1 basis set. Energy reference was set to vacuum according to the protocol reported by Butler et al. ${ }^{[58]}$ Crystal structures, crystal orbitals and electron density differences were plotted by means of VESTA (version 3.4.6) software. ${ }^{[59]}$ NonCovalent Index (NCI) calculations were performed under the NCIPLOT-3.0 software ${ }^{[60,61]}$ using the default PROMOLECULAR atomic densities, and density and gradient thresholds. The intermolecular contribution to the $\mathrm{NCl}$ surfaces was calculated by means of the INTERMOLECULAR keyword, and the VMD-1.9.3 software ${ }^{[62]}$ was employed for graphical display. Molecular DFT calculations were performed for the $\mathbf{C}_{60} @ T T F T B$ system using the Gaussian-16.A03 suite of packages. ${ }^{[63]}$ Hydrogen atoms were added in the terminal carboxylate groups for charge neutrality. Interaction energies were calculated for the previously PBC-optimized crystal structures as the energy difference between the dimer and the constituting monomers. The hybrid B3LYP[64] with the Grimme's D3 dispersion correction ${ }^{[65]}$ (B3LYP-D3) was employed along with the $6-31 G^{* *}$ basis set and half of the counterpoise correction $(\mathrm{CP})$. Time-dependent 
DFT (TDDFT) calculations were performed using the coulomb-attenuating CAMB3LYP approach ${ }^{[6]}$ with the $6-31 \mathrm{G}^{* *}$ basis set for the lowest-lying excited states. Excitation energies were convoluted with Gaussian functions with full-width-at-halfmaximum (FWHM) of $0.2 \mathrm{eV}$. Charge transfer was evaluated as the accumulated natural population analysis (NPA ${ }^{[67]}$ charges on each moiety. Molecular orbitals were represented by means of the Chemcraft 1.7 software. ${ }^{[68]}$

Electrical measurements. Pressed pellets ( $P \approx 5$ US tons) were cut in rectangular shapes and contacted with silver conductive paint (RS 123-9911) and platinum wires (Goodfellow, 99.99\%, $25 \mu \mathrm{m}$ of diameter) in a 4 probe configuration (Figures S2-S3). The geometrical factors (thickness, width and length were measured using an optical microscope (width and length were determined from the top view, Figures S2-3a, and the thickness from the lateral one, Figures S2-3b). I-V curves were measured with a Keithley 6517B electrometer for ultra-high resistance/ultra-low current measurements in a two-probe configuration, i.e., applying a voltage bias between two leads and measuring the current between them. 


\section{Supporting Information}

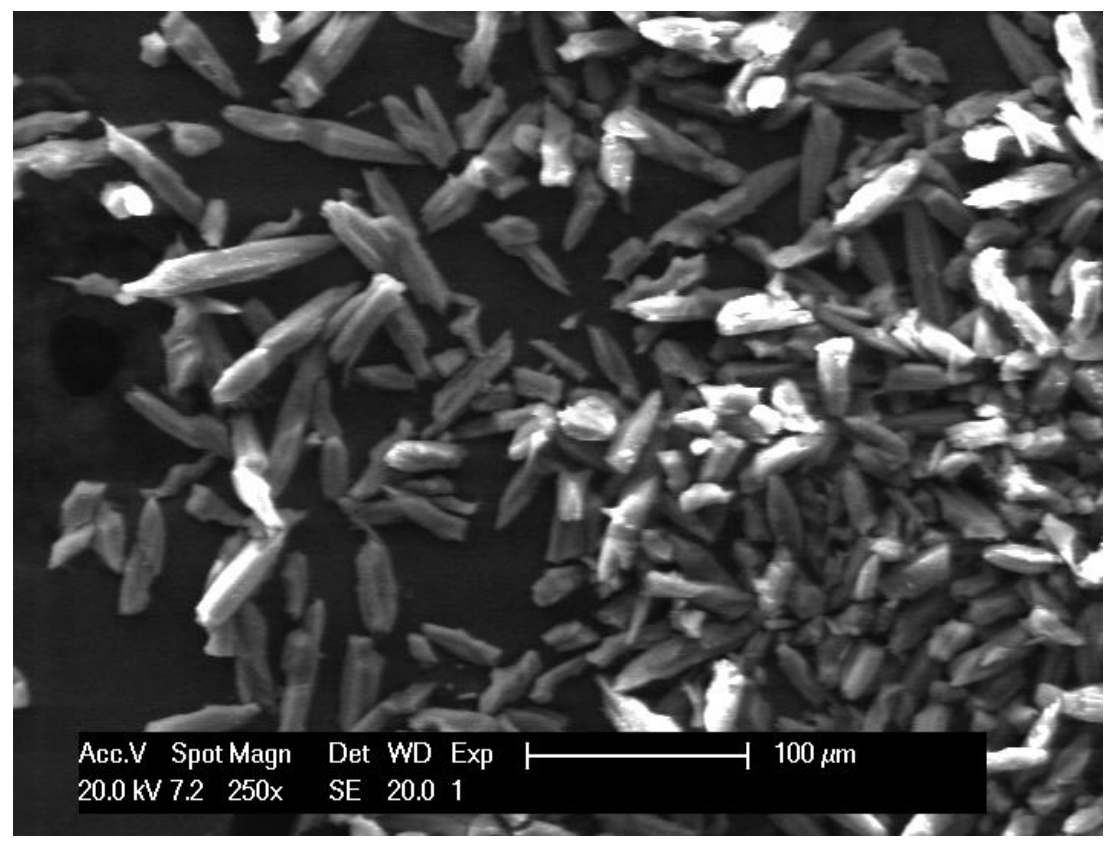

Figure S1: SEM image of $\mathbf{C}_{60} @ M U V-2$.

a

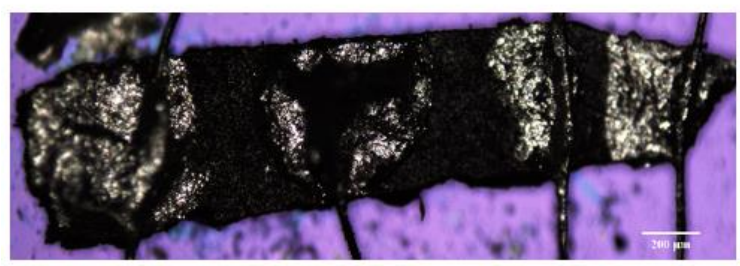

b

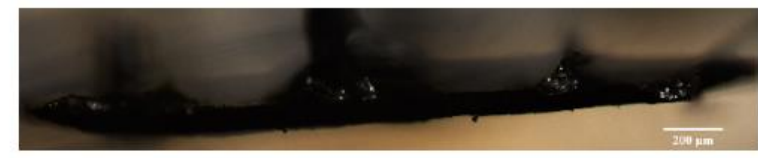

Figure S2: Top view (a) and lateral view (b) for MUV-2 electrical measurements.

a

b
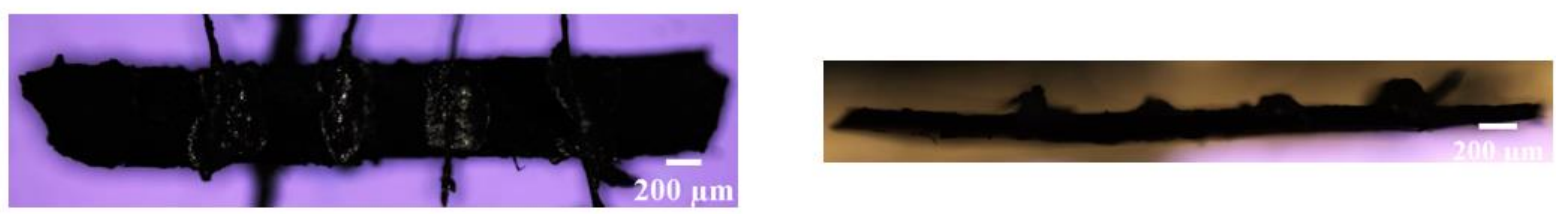

Figure S3: Top view (a) and lateral view (b) for $\mathbf{C}_{60} @ \mathbf{M U V - 2}$ electrical measurements. 
Table S1: Charge transfer (in electron charge units, e) from the electron-donor TTFTB ligand to the electron-acceptor $\mathrm{C}_{60}$ guest in the ground state (GS) and the charge-transfer $\mathrm{S}_{1}$ state $(\mathrm{CT})$ of $\mathrm{C}_{60} @$ $@$ TTFTB as a function of the distance between centroids ( $d$ ). Data corresponding to the minimum-energy geometry is in bold.

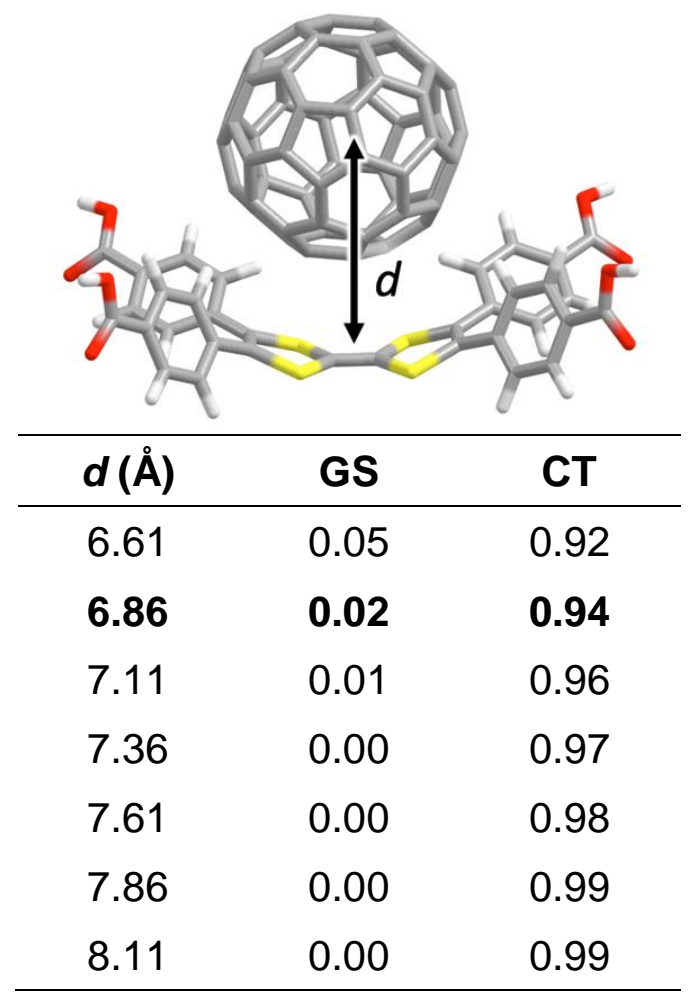


Table S2: Intense lowest-lying singlet excited states of TTFTB ligand and $\mathrm{C}_{60}$ guest, calculated at the CAM-B3LYP/6-31G** level of theory. The energy $(E)$, oscillator strength $(f)$, major monoexcitation, and description of each state are included.

\begin{tabular}{|c|c|c|c|c|c|}
\hline State & $E(\mathrm{eV})$ & $E(\mathrm{~nm})$ & $f$ & Monoexcitation & Description \\
\hline \multicolumn{6}{|c|}{ TTFTB } \\
\hline $\mathrm{S}_{1}$ & 2.907 & 426.4 & 0.0760 & $H \rightarrow L(68 \%)$ & TTF $\rightarrow$ peripheral \\
\hline $\mathrm{S}_{3}$ & 3.486 & 355.7 & 0.1043 & $H \rightarrow L+4(58 \%)$ & TTF \\
\hline $\mathrm{S}_{6}$ & 4.261 & 291.0 & 0.4840 & $H \rightarrow L+3(52 \%)$ & TTF $\rightarrow$ peripheral \\
\hline $\mathrm{S}_{8}$ & 4.430 & 279.9 & 0.5490 & $\mathrm{H}-1 \rightarrow \mathrm{L}+1(51 \%)$ & TTF $\rightarrow$ peripheral \\
\hline $\mathrm{S}_{21}$ & 5.217 & 237.7 & 0.4389 & $\mathrm{H}-3 \rightarrow \mathrm{L}(15 \%)$ & TTF $\rightarrow$ peripheral \\
\hline $\mathrm{S}_{54}$ & 6.623 & 187.2 & 0.7300 & $\mathrm{H}-4 \rightarrow \mathrm{L}+5$ (13\%) & TTF + peripheral \\
\hline \multicolumn{6}{|c|}{$\mathrm{C}_{60}$} \\
\hline $\mathrm{S}_{37}$ & 4.309 & 287.7 & 0.2196 & $\mathrm{H}-2 \rightarrow \mathrm{L}+3(18 \%)$ & - \\
\hline $\mathrm{S}_{38}$ & 4.312 & 287.6 & 0.2196 & $\mathrm{H}-3 \rightarrow \mathrm{L}+5(21 \%)$ & - \\
\hline $\mathrm{S}_{39}$ & 4.315 & 287.3 & 0.2206 & $\mathrm{H}-4 \rightarrow \mathrm{L}+5(31 \%)$ & - \\
\hline$S_{52}$ & 4.703 & 263.7 & 0.1192 & $H-6 \rightarrow$ L+2 (19\%) & - \\
\hline $\mathrm{S}_{53}$ & 4.704 & 263.6 & 0.1181 & $\mathrm{H}-6 \rightarrow \mathrm{L}+1(21 \%)$ & - \\
\hline $\mathrm{S}_{54}$ & 4.706 & 263.5 & 0.1133 & H-8 $\rightarrow$ L (17\%) & - \\
\hline
\end{tabular}


Table S3: Evolution of $\mathrm{S}_{1} \mathrm{CT}$ excitation for $\mathbf{C}_{60} @$ TTFTB as a function of distance $d$ (as defined in Table S1) and the TTF boat dihedral angle $(\theta)$. Data corresponding to the minimum-energy geometry is in bold.

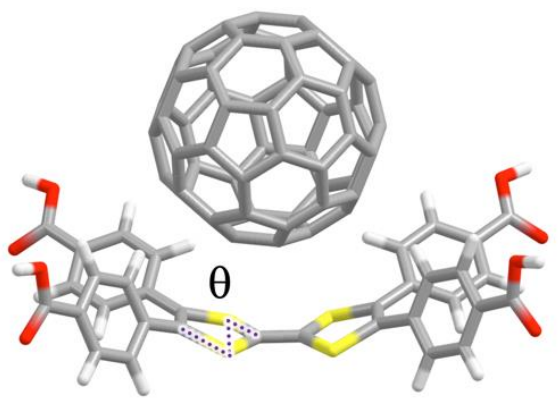

\begin{tabular}{cccc}
\hline $\boldsymbol{d}(\AA)$ & $E(\mathrm{eV})$ & $E(\mathrm{~nm})$ & $\boldsymbol{f}$ \\
\hline 6.61 & 2.146 & 577.8 & 0.0284 \\
$\mathbf{6 . 8 6}$ & $\mathbf{2 . 1 4 3}$ & $\mathbf{5 7 8 . 6}$ & $\mathbf{0 . 0 1 7 8}$ \\
7.11 & 2.167 & 572.1 & 0.0103 \\
7.36 & 2.209 & 561.3 & 0.0054 \\
7.61 & 2.258 & 549.0 & 0.0027 \\
7.86 & 2.310 & 536.8 & 0.0012 \\
8.11 & 2.360 & 525.4 & 0.0005 \\
\hline $\boldsymbol{\theta}(\stackrel{\circ}{)})$ & $\boldsymbol{E}(\mathrm{eV})$ & $\boldsymbol{E ( n m )}$ & $\boldsymbol{f}$ \\
\hline $\mathbf{1 6 2}$ & $\mathbf{2 . 1 4 3}$ & $\mathbf{5 7 8 . 6}$ & $\mathbf{0 . 0 1 7 8}$ \\
171 & 2.044 & 606.7 & 0.0131 \\
180 & 2.025 & 612.3 & 0.0079 \\
\hline
\end{tabular}


Supporting Information File 1:

File Name: $\mathbf{C}_{60} @ M U V-2$ confA

File Format: CIF

Title: Simulated structure of $\mathrm{C}_{60} @ M U V-2$ conformation A

Supporting Information File 2:

File Name: $\mathbf{C}_{60} @ M U V-2$ confB

File Format: CIF

Title: Simulated structure of $\mathrm{C}_{60} @ M U V-2$ conformation B 


\section{Acknowledgements}

This work has been supported by the European Union (ERC-2016-CoG 724681-S-CAGE) and the Spanish MICINN (CTQ2017-89528-P). G.M.E. and M.S. thank MICINN for a Ramón y Cajal and a Juan de la Cierva-Formación fellowships, respectively. J.C. acknowledges the Generalitat Valenciana for the postdoctoral APOSTD/2017/081 fellowship. Via our membership of the UK's HEC Materials Chemistry Consortium, which is funded by EPSRC (EP/L000202), this work used the ARCHER UK National Supercomputing Service (http://www.archer.ac.uk).

\section{References}

1. H. C. Zhou, J. R. Long, O. M. Yaghi, Chem. Rev., 2012, 112, 673-674.

2. G. Maurin, C. Serre, A. Cooper, G. Férey, Chem. Soc. Rev., 2017, 46, 31043107.

3. H. Furukawa, K. E. Cordova, M. O'Keeffe, O. M. Yaghi, Science, 2013, 341, 1230444 .

4. A. G. Slater, A. I. Cooper, Science, 2015, 348, aaa8075.

5. S. M. J. Rogge, A. Bavykina, J. Hajek, H. Garcia, A. I. Olivos-Suarez, A.

Sepúlveda-Escribano, A. Vimont, G. Clet, P. Bazin, F. Kapteijn, M. Daturi, E. V. Ramos-Fernandez, F. X. Llabrés i Xamena, V. Van Speybroeck, Chem. Soc. Rev., 2017, 46, 3134-3184.

6. L. Sun, M. G. Campbell, M. Dincă, Angew. Chem. Int. Ed., 2016, 55, 35663579 .

7. I. Stassen, N. Burtch, A. Talin, P. Falcaro, M. Allendorf, R. Ameloot, Chem. Soc. Rev., 2017, 46, 3185-3241.

8. T. Bein, D. D. Medina, Isr. J. Chem., 2018, 4, 1089-1101. 
9. M. G. Campbell, D. Sheberla, S. F. Liu, T. M. Swager, M. Dincă, Angew. Chem. Int. Ed., 2015, 54, 4349-4352.

10. G. Wu, J. Huang, Y. Zang, J. He, G. Xu, J. Am. Chem. Soc., 2017, 139, 13601363.

11. D. Sheberla, J. C. Bachman, J. S. Elias, C. Sun, Y. Shao-horn, Nat. Mat., 2017, $16,220-224$.

12. A. A. Talin, A. Centrone, A. C. Ford, M. E. Foster, V. Stavila, P. Haney, R. A. Kinney, V. Szalai, F. El Gabaly, H. P. Yoon, F. Léonard, M. D. Allendorf, Science, 2014, 343, 66-69.

13. S. K. Bhardwaj, N. Bhardwaj, R. Kaur, J. Mehta, A. L. Sharma, K. Kim, A. Deep, J. Mater. Chem. A, 2018, 6, 14992-15009.

14. C. Kung, K. Otake, C. T. Buru, S. Goswami, Y. Cui, J. T. Hupp, A. M. Spokoyny, O. K. Farha, J. Am. Chem. Soc., 2018, 140, 3871-3875.

15. D. M. D'Alessandro, Chem. Commun., 2016, 52, 8957-8971.

16. C. W. Kung, T. C. Wang, J. E. Mondloch, D. Fairen-Jimenez, D. M. Gardner, W. Bury, J. M. Klingsporn, J. C. Barnes, R. Van Duyne, J. F. Stoddart, M. R. Wasielewski, O. K. Farha, J. T. Hupp, Chem. Mater., 2013, 25, 5012-5017.

17. Q. Chen, J. Sun, P. Li, I. Hod, P. Z. Moghadam, Z. S. Kean, R. Q. Snurr, J. T. Hupp, O. K. Farha, J. F. Stoddart, J. Am. Chem. Soc., 2016, 138, 1424214245.

18. J. Calbo, M. J. Golomb, A. Walsh, J. Mater. Chem. A, 2019, DOI 10.1039/C9TA04680A.

19. A. Van Wyk, T. Smith, J. Park, P. Deria, J. Am. Chem. Soc., 2018, 140, 27562760.

20. C. Hua, P. W. Doheny, B. Ding, B. Chan, M. Yu, C. J. Kepert, D. M. D'Alessandro, J. Am. Chem. Soc., 2018, 140, 6622-6630. 
21. H. Miyasaka, Acc. Chem. Res., 2013, 46, 248-257.

22. H. W. Kroto, J. R. Heath, S. C. O'Brien, R. F. Curl, R. E. Smalley, Nature, $1985,318,162-163$.

23. F. Giacalone, N. Martín, Chem. Rev., 2006, 106, 5136-5190.

24. N. Martín, Chem. Commun., 2006, 2093-2104.

25. A. Zieleniewska, F. Lodermeyer, A. Roth, D. M. Guldi, Chem. Soc. Rev., 2018, $47,702-714$.

26. H. Meng, C. Wang, T. Wang, Gen. Chem., 2018, 4, 180019.

27. Y. Inokuma, T. Arai, M. Fujita, Nat. Chem., 2010, 2, 780-783.

28. C. García-Simón, M. Garcia-Borràs, L. Gómez, T. Parella, S. Osuna, J. Juanhuix, I. Imaz, D. Maspoch, M. Costas, X. Ribas, Nat. Commun., 2014, 5, 5557.

29. C. García-Simón, M. Costas, X. Ribas, Chem. Soc. Rev., 2016, 45, 40-62.

30. Y. Feng, T. Wang, Y. Li, J. Li, J. Wu, B. Wu, L. Jiang, C. Wang, J. Am. Chem. Soc., 2015, 137, 15055-15060.

31. H. Meng, C. Zhao, M. Nie, C. Wang, T. Wang, J. Phys. Chem. C, 2019, 123, 6265-6269.

32. X. Liu, M. Kozlowska, T. Okkali, D. Wagner, T. Higashino, G. Brenner-Weiß, S. M. Marschner, Z. Fu, Q. Zhang, H. Imahori, S. Bräse, W. Wenzel, C. Wöll, L. Heinke, Angew. Chem. Int. Ed., 2019, 58, 1-7.

33. S. Goswami, D. Ray, K. Otake, C. Kung, S. J. Garibay, T. Islamoglu, A. Atilgan, Y. Cui, C. J. Cramer, O. K. Farha, J. T. Hupp, Chem. Sci. 2018, 9, 4477-4482.

34. J. L. Segura, N. Martn, Angew. Chem. Int. Ed. 2001, 40, 1372-1409.

35. N. Martín, Chem. Commun., 2013, 49, 7025-7027.

36. A. Jana, S. Bähring, M. Ishida, S. Goeb, D. Canevet, M. Sallé, J. O. Jeppesen, J. L. Sessler, Chem. Soc. Rev., 2018, 47, 5614-5645. 
37. J. M. Spruell, A. Coskun, D. C. Friedman, R. S. Forgan, A. A. Sarjeant, A. Trabolsi, A. C. Fahrenbach, G. Barin, W. F. Paxton, S. K. Dey, et al., Nat. Chem., 2010, 2, 870-879.

38. M. Frasconi, T. Kikuchi, D. Cao, Y. Wu, W. G. Liu, S. M. Dyar, G. Barin, A. A. Sarjeant, C. L. Stern, R. Carmieli, C. Wang, M. R. Wasielewski, W. A. Goddard III, J. F. Stoddart, J. Am. Chem. Soc., 2014, 136, 11011-11026.

39. E. M. Pérez, L. Sánchez, G. Fernández, N. Martín, J. Am. Chem. Soc., 2006, $128,7172-7173$.

40. E. M. Pérez, M. Sierra, L. Sánchez, M. R. Torres, R. Viruela, P. M. Viruela, E. Ortí, N. Martín, Angew. Chem. Int. Ed., 2007, 46, 1847-1851.

41. E. M. Pérez, N. Martín, Chem. Soc. Rev., 2008, 37, 1512-1519.

42. D. Canevet, E. M. Pérez, N. Martín, Angew. Chem. Int. Ed. 2011, 50, 92489259.

43. S. Goeb, S. Bivaud, P. I. Dron, J. Y. Balandier, M. Chas, M. Sallé, Chem. Commun. 2012, 48, 3106-3108.

44. M. Gallego, J. Calbo, J. Aragó, R. M. Krickcalderon, F. H. Liquido, T. Iwamoto, A. K. Greene, E. A. Jackson, E. M. Pérez, E. Ortí, D. M. Guldi, L. T. Scott, N. Martín, Angew. Chem. Int. Ed., 2014, 53, 2170-2175.

45. H.-Y. Wang, L. Cui, J.-Z. Xie, C. F. Leong, D. M. D'Alessandro, J.-L. Zuo, Coord. Chem. Rev., 2017, 345, 342-361.

46. S. S. Park, E. R. Hontz, L. Sun, C. H. Hendon, A. Walsh, T. Van Voorhis, M. Dincă, J. Am. Chem. Soc., 2015, 137, 1774-1777.

47. J. Su, S. Yuan, H.-Y. Wang, L. Huang, J.-Y. Ge, E. Joseph, J. Qin, T. Cagin, J.L. Zuo, H.-C. Zhou, Nat. Commun. 2017, 8, 2008.

48. H.-Y. Wang, J.-Y. Ge, C. Hua, C.-Q. Jiao, Y. Wu, C. F. Leong, D. M. D’Alessandro, T. Liu, J.-L. Zuo, Angew. Chem. Int. Ed., 2017, 56, 5465 -5470. 
49. M. Souto, A. Santiago-Portillo, M. Palomino, I. J. Vitórica-Yrezábal, B. J. C. Vieira, J. C. Waerenborgh, S. Valencia, S. Navalón, F. Rey, H. García, G. Mínguez Espallargas, Chem. Sci., 2018, 9, 2413-2418.

50. M. Souto, J. Romero, J. Calbo, I. J. Vitorica-Yrezabal, J. L. Zafra, J. Casado Cordón, E. Ortí, A. Walsh, G. Mínguez Espallargas, J. Am. Chem. Soc., 2018, 140, 10562-10569.

51. M. Vicent-Morales, I. J. Vitórica-Yrezábal, M. Souto, G. Mínguez Espallargas, CrystEngComm 2019, 21, 3031-3035.

52. H. K. Chae, D. Y. Siberio-Pérez, J. Kim, Y. Go, M. Eddaoudi, A. J. Matzger, M. O’Keeffe, O. M. Yaghi, Nature, 2004, 427, 523-527.

53. H. Li, M. R. Hill, R. Huang, C. Doblin, S. Lim, A. J. Hill, R. Babarao, P. Falcaro, Chem. Commun., 2016, 52, 5973-5976.

54. V. Blum, R. Gehrke, F. Hanke, P. Havu, V. Havu, X. Ren, K. Reuter, M. Scheffler, Comput. Phys. Commun., 2009, 180, 2175-2196.

55. J. P. Perdew, A. Ruzsinszky, G. I. Csonka, O. A. Vydrov, G. E. Scuseria, L. A. Constantin, X. Zhou, K. Burke., Phys. Rev. Lett., 2008, 100, 136406.

56. A. Tkatchenko, M. Scheffler, Phys. Rev. Lett., 2009, 102, 73005.

57. J. Heyd, G. E. Scuseria, M. Ernzerhof, J. Chem. Phys., 2003, 118, 8207-8215.

58. K. T. Butler, C. H. Hendon, A. Walsh, J. Am. Chem. Soc., 2014, 136, $2703-$ 2706.

59. K. Momma, F. Izumi, J. Appl. Crystallogr., 2011, 44, 1272-1276.

60. S. Keinan, J. Contreras-García, E. R. Johnson, W. Yang, P. Mori-Sánchez, A. J. Cohen, J. Am. Chem. Soc., 2010, 132, 6498-6506.

61. J. Contreras-García, E. R. Johnson, S. Keinan, R. Chaudret, J. P. Piquemal, D. N. Beratan, W. Yang, J. Chem. Theory Comput., 2011, 7, 625-632.

62. W. Humphrey, A. Dalke, K. Schulten, J. Molec. Graph., 1996, 14, 33-38. 
63. Gaussian 16, Revision A.03, M. J. Frisch, G. W. Trucks, H. B. Schlegel, G. E. Scuseria, M. A. Robb, J. R. Cheeseman, G. Scalmani, V. Barone, G. A. Petersson, H. Nakatsuji, X. Li, M. Caricato, A. V. Marenich, J. Bloino, B. G. Janesko, R. Gomperts, B. Mennucci, H. P. Hratchian, J. V. Ortiz, A. F. Izmaylov, J. L. Sonnenberg, D. Williams-Young, F. Ding, F. Lipparini, F. Egidi, J. Goings, B. Peng, A. Petrone, T. Henderson, D. Ranasinghe, V. G. Zakrzewski, J. Gao, N. Rega, G. Zheng, W. Liang, M. Hada, M. Ehara, K. Toyota, R. Fukuda, J. Hasegawa, M. Ishida, T. Nakajima, Y. Honda, O. Kitao, H. Nakai, T. Vreven, K. Throssell, J. A. Montgomery, Jr., J. E. Peralta, F. Ogliaro, M. J. Bearpark, J. J. Heyd, E. N. Brothers, K. N. Kudin, V. N. Staroverov, T. A. Keith, R. Kobayashi, J. Normand, K. Raghavachari, A. P. Rendell, J. C. Burant, S. S. Iyengar, J. Tomasi, M. Cossi, J. M. Millam, M. Klene, C. Adamo, R. Cammi, J. W. Ochterski, R. L. Martin, K. Morokuma, O. Farkas, J. B. Foresman, and D. J. Fox, Gaussian, Inc., Wallingford CT, 2016.

64. A. D. Becke, J. Chem. Phys., 1993, 98, 5648-52.

65. S. Grimme, S. Ehrlich, L. Goerigk, J. Comp. Chem., 2011, 32, 1456-1465.

66. T. Yanai, D. P. Tew, N. C. Handy, Chem. Phys. Lett. 2004, 393, 51-57.

67. F. Weinhold, J. E. Carpenter, in (Eds.: R. Naaman, Z. Vager), 1988, pp. 227236.

68. Chemcraft - graphical software for visualization of quantum chemistry computations. https://www.chemcraftprog.com 
